\title{
Una caracterización de la pobreza femenina en México conforme al modelo colectivo de hogar*
}

\author{
Katya Rodríguez Gómez**
}

En este texto se examinan, tal como exhorta la CEPAL (2004), la evidencia que existe acerca de la pobreza femenina en México y sus causas, tomando como base la Encuesta Nacional de Ingreso y Gasto de los Hogares 2008. Se mide la pobreza tomando en cuenta una consideración más realista acerca de los recursos que las mujeres tienen a su disposición, para lo cual se aplica el modelo colectivo de hogar. Se exponen estadísticas descriptivas que muestran la relación de la pobreza femenina y masculina con otras divisiones sociales. Posteriormente se construye un modelo para determinar el peso específico en la explicación de la pobreza femenina de los tres mecanismos que distribuyen recursos en la sociedad: el mercado, el Estado y la familia.

Palabras clave: pobreza, mujeres, género, modelo colectivo, México. Fecha de recepción: 25 de abril de 2012.

Fecha de aceptación: 3 de abril de 2013.

\section{A Characterization of Female Poverty in Mexico Based on the Collective Household Model}

As requested by ECLAC (2004), this text examines the evidence on female poverty in Mexico and its cause, based on the National Household Income and Expenditure Survey 2008. Poverty is measured by taking into account a more realistic consideration about the resources available to women, for which the collective household model is applied. The article provides descriptive statistics showing the link between male to female poverty and other social divisions. The author subsequently constructs a model to determine the specific importance in the explanation of female poverty of the three mechanisms that distribute resources in society: the market, the state and the family.

Key words: poverty, women, gender, collective model, Mexico.

\footnotetext{
* Se agradecen los comentarios de los dos dictaminadores anónimos que contribuyeron con pertinencia al enriquecimiento del documento.

** Departamento de Gestión Pública y Desarrollo, División de Ciencias Sociales y Humanidades, campus León, Universidad de Guanajuato. Correo electrónico: <katyarg@ yahoo.com>y<katyarogz@ugto.mx>.
} 


\section{Introducción}

Desde 1995, año en el que tuvo lugar la Cuarta Conferencia Mundial sobre la Mujer, organizada por las Naciones Unidas, se asumió la idea de que la feminización de la pobreza era un problema en el mundo y había que tomar acciones para erradicarla. El concepto dominó por un tiempo la visión sobre la pobreza femenina tanto en los trabajos académicos como en los de organismos internacionales (Graham, 1996; Asian Development Bank, 2003). Sin embargo, posteriormente se ha demostrado que tal concepto no necesariamente es útil para dar cuenta de la realidad de la pobreza femenina (Chant, 2007; Medeiros y Costa, 2010; Sen, 2010).

El cuestionamiento se basa en dos puntos fundamentales. Uno es el concepto mismo, el otro es el tipo de evidencia disponible. Respecto al concepto se ha señalado que no resulta útil para dar cuenta de la realidad de la pobreza femenina por varias razones. En primer lugar, la feminización implica dinamismo, sin embargo no se ha probado que la situación de las mujeres vaya empeorando con el tiempo (Chant, 2007). También se ha tendido a relacionar la idea de la feminización con el hecho de que los hogares encabezados por mujeres son más pobres. No obstante, dichos hogares encabezados por mujeres pueden encontrarse con frecuencia en una mejor situación, como resultado de la búsqueda de equilibrio entre un menor ingreso y un mayor bienestar (Chant, 1997). Además en muchos países se encuentra que los hogares encabezados por mujeres son menos pobres que los encabezados por hombres (Nauckhoff, 2004). Por otro lado, la evidencia de que existen más mujeres pobres que hombres pobres ha sido difícil de probar debido a los problemas en las fuentes de información disponibles. Ello se debe a que la pobreza se mide asumiendo que el ingreso total de los hogares de divide por igual entre todos sus miembros, lo que resulta inexacto. Existe carencia de bases de datos que den cuenta de la distribución de los recursos en el interior de los hogares. Por tanto, la idea de la feminización de la pobreza ha sido difícil de sostener con evidencia empírica sólida (Johnsson-Latham, 2004; Molyneux, 2006b). El caso particular de México, del que se ocupa este artículo, no ha resultado diferente de lo anterior. Varios autores han mostrado evidencia cuantitativa que apunta a que no existe feminización de la pobreza (Villareal y Shin, 2008; Damián, 2003, 2008, 2011).

Más recientemente, ha habido un llamado por parte de la Comisión Económica para América Latina y el Caribe (CEPAL, 2004: 82) que urge 
a interpretar las características de la pobreza femenina y sus causas, como una de las principales prioridades para América Latina y el Caribe. El objetivo que persigue la CEPAL es que más allá de lugares comunes, la evidencia sea realmente pertinente para poder diseñar políticas apropiadas que mejoren la situación de las mujeres respecto a la pobreza.

En el tenor de esta discusión, el presente artículo resulta una continuación de un trabajo previo (Rodríguez, 2012) en donde se intentaba responder a la pregunta sobre si existe feminización de la pobreza en México. Para ello se atendía a los dos grandes cuestionamientos que se mencionaron arriba. En primer lugar se examinaban los tres elementos que han sido asociados a la feminización (que las mujeres son la mayoría de los pobres, que existe una tendencia dinámica al incremento de la pobreza femenina y que los hogares encabezados por mujeres son más pobres) con evidencia calculada de manera convencional, es decir, asumiendo que los recursos que perciben todos los miembros del hogar se comparten por igual en su interior (modelo unitario de hogar). Aplicando el modelo unitario no se cumplen ninguna de las tres condiciones que se asocian al concepto de feminización de la pobreza, por lo que desde ahí es posible concluir sin mayor problema que no existe feminización de la pobreza en México. El segundo cuestionamiento ha estado relacionado con la manera en que se mide la pobreza. Es por ello que en el artículo, para proseguir con la discusión, se aplicó un modelo diferente de distribución de recursos en el hogar: el modelo colectivo, que asume que no todos los recursos son compartidos por igual, sino que los miembros que perciben ingresos retienen para sí una cantidad de los mismos. En el artículo se examinaban las implicaciones de este cambio, que coincide mejor con lo que la literatura especializada ha descrito que sucede en el interior de los hogares. Al aplicarlo se usaron diversas asunciones respecto a la cantidad de recursos retenida por los miembros que perciben ingresos. Con todas ellas (desde la más baja hasta la más alta) se encontró que puede apreciarse claramente una brecha de género en la pobreza. Es decir, la pobreza femenina es mayor que la masculina, y la pobreza femenina es mucho más aguda en los hogares encabezados por hombres que en los encabezados por mujeres. El artículo concluía que si bien no puede hablarse de feminización de la pobreza en el caso de México, porque no se verifica un proceso dinámico hacia un empeoramiento en el tiempo de la situación de las mujeres, ni existe una peor situación en los hogares con jefatura feme- 
nina, sí es claro que la tasa de pobreza femenina es mayor que la masculina. Por ello, usar el concepto de feminización tiende a oscurecer, más que a aclarar la situación de las mujeres. Lo que resulta más útil e importante es caracterizar la situación de las mujeres respecto a la pobreza.

El presente trabajo se abocará, en consecuencia, a realizar de manera detallada una caracterización de la pobreza femenina en México y a analizar algunas de sus causas. Para ello se usa la Encuesta Nacional de Ingreso y Gasto de los Hogares (ENIGH) 2008. ${ }^{1}$ Dicha encuesta resulta particularmente atractiva porque presenta nuevas características tales como que por primera vez se incorporan las recomendaciones de la Organización Internacional del Trabajo (OIT) y del Grupo de Canberra para la colección de estadísticas de ingreso y gasto con estándares y comparabilidad internacionales. Asimismo, esta encuesta tiene una muestra ampliada, dado que se le agrega el Módulo de Condiciones Socioeconómicas (MCS) por encargo del Consejo Nacional de Evaluación de la Política Social (Coneval), para poder contar con información suficiente para medir la pobreza por entidad federativa. Otro punto importante es que se incorporaron nuevas variables a la información que tradicionalmente recogía la ENIGH, tales como hablar lengua indígena, las características del acceso a la alimentación, la condición de discapacidad, etc., que ampliaron sustancialmente la calidad de la información que actualmente maneja la ENIGH. En este estudio se aprovechan estas nuevas características usando los datos que proceden de la base ampliada de la ENIGH-MCS en su nueva construcción. ${ }^{2}$

En la siguiente sección se abordará la pobreza femenina en términos de carencia de ingreso; por lo pronto adelantamos dos cuestiones importantes: en la sociedad contemporánea el ingreso resulta fundamental como vía de acceso a prácticamente todos los satisfactores, y además la carencia de ingreso está altamente correlacionada con otras manifestaciones de la pobreza como el estrés emocional, los problemas de salud, la discriminación y la exclusión social en general (Razavi y Staab, 2010). Es importante hacer notar que si bien hay otras maneras de entender la cuestión de género, este artículo está particularmente

${ }^{1}$ En el momento de iniciar esta investigación la última base de datos disponible era la ENIGH 2008, dado que los resultados de la ENIGH 2010 se publicaron en julio de 2011.

${ }^{2}$ La nueva construcción incorpora las características mencionadas; conviene diferenciarla de la ENIGH 2008 en su modalidad tradicional, que también fue publicada para lograr compatibilidad con las ENIGH anteriores. 
interesado en abordar el tema del acceso a los recursos por parte de las mujeres y los procesos por los cuales se les discrimina en la sociedad para obtener dichos recursos.

Para llevar adelante esta tarea se intentará superar una de las principales críticas que ha recibido el estudio de la pobreza femenina, que ya se mencionó. Si bien la pobreza muestra diferencias de género en su incidencia, sus causas y sus efectos, los datos que la miden tienden a ser inadecuados. Ello porque lo que realmente están midiendo es el número de individuos, tanto mujeres como hombres, que viven en hogares en pobreza, asumiendo que todos los miembros del hogar comparten el ingreso de igual manera (Lister, 2010: 167). Asumir lo anterior resulta equívoco dada la gran cantidad de estudios que muestran que el ingreso no se comparte de manera justa en la familia y que esto generalmente suele ir en detrimento de las mujeres (Pahl, 1989; Heintz, 2010; Razavi y Staab, 2010).

Por lo anterior, resulta muy importante lograr medir la pobreza de las mujeres en términos de los ingresos que realmente tienen a su disposición (Lister, 2010: 171). Sin embargo, ello conlleva una gran dificultad metodológica por la carencia de bases de datos que permitan medir los ingresos que realmente son recibidos por los individuos dentro de los hogares.

Para superar ese obstáculo, el artículo aplica el modelo colectivo de hogar, que asume que no todos los ingresos dentro del hogar son puestos en una bolsa común, sino que una parte de éstos la retiene para su consumo personal quien los recibe (Quisumbing, 2010; Falkingham y Baschieri, 2009, 2010). De esta manera los miembros del hogar que reciben recursos tienen una mejor situación ante la pobreza que los que no los reciben. Este modelo permite dar cuenta de algunas de las diferencias de género que esconde el modelo convencional de análisis de la pobreza (unitario).

Por tanto, en el artículo se proponen dos objetivos fundamentales: interpretar la evidencia sobre la pobreza femenina en México mediante el examen de sus causas específicas, y realizar dicha caracterización desde el modelo colectivo de hogar. La operacionalización de la pobreza es realizada usando la Línea de Bienestar de Coneval, ${ }^{3}$ que declara ser la cantidad de ingreso necesaria para identificar a la población que, si está por encima de ella, puede satisfacer sus necesidades esen-

${ }^{3}$ Para conocer una explicación acerca de por qué no se usa la medición multidimensional de la pobreza véase el apartado "Sobre el cálculo del ingreso y la línea de pobreza”. 
ciales. No obstante, el ejercicio del cálculo de las tasas de pobreza femeninas y masculinas en México se realiza también usando ciertos estimados que permiten modificar la Línea de Bienestar de Coneval, para incluir, entre otros elementos, el costo de generar el ingreso para aquellas personas que estudian o trabajan fuera del hogar. Ello porque la Línea de Bienestar original no considera del todo estos costos y porque este aspecto resulta particularmente importante si se aplica el modelo colectivo, debido a que este modelo asume una retención de ingreso para consumo personal por parte de los perceptores. El objetivo de aplicar estas modificaciones a la Línea de Bienestar de Coneval es conocer si considerando lo anterior se sostiene la evidencia sobre las brechas de pobreza entre hombres y mujeres al usar el modelo colectivo.

Para cumplir con sus objetivos el artículo se divide en varias partes, incluyendo esta introducción. La segunda parte justifica la importancia del ingreso para abordar la pobreza femenina y expone la relación entre las tres instituciones sociales que determinan el acceso al ingreso: el mercado, el Estado y la familia. Ello sirve de guía para explicar los distintos factores sociales que pueden contribuir a crear la pobreza femenina. La tercera parte discierne las diferencias entre el modelo unitario y el modelo colectivo para medir la pobreza; y muestra las evidencias que han encontrado algunos estudios anteriores realizados en México sobre la pobreza femenina usando ambos modelos. La cuarta parte expone la metodología para el cómputo del ingreso y el cálculo de la pobreza dentro del modelo colectivo usando tanto la Línea de Bienestar de Coneval como la modificación que se hace de ésta, y se exponen los resultados de las tasas de pobreza femenina y masculina conforme a ambos supuestos. En la quinta parte se emprende el análisis sobre la pobreza femenina, exponiendo estadísticas descriptivas que muestran la relación de la pobreza femenina y masculina con otras divisiones sociales para comparar la variación en la brecha de género cuando se pasa del modelo unitario al colectivo y se establece un primer orden de hallazgos. La sexta parte construye un modelo que permite explicar el peso específico de los factores relacionados con las tres instituciones que distribuyen recursos en la sociedad y cómo éstos determinan la pobreza femenina. Con base en los hallazgos del artículo, la séptima parte menciona explícitamente las causas más importantes de la pobreza femenina que pueden deducirse de este ejercicio. Por último, las conclusiones resaltan los principales aportes del artículo. 


\section{La importancia del ingreso y el papel del mercado, el Estado y el hogar en la pobreza femenina}

En este caso se abordará la pobreza femenina valiéndose del ingreso como criterio para definirla y medirla, sin ignorar que diversos autores advierten acerca de las limitaciones de basarse únicamente en el ingreso y en otros aspectos mesurables para examinarla, y reconociendo la necesidad de incorporar enfoques que revelen la subjetividad de las mujeres y la multidimensionalidad de la pobreza (Chant, 2007, 2009; Moghadam, 1997). Algunos estudios cualitativos acerca de la manera en que las mujeres perciben la pobreza (Chant, 2009) demuestran que no necesariamente el punto más apremiante que resaltan es la falta de ingresos; para ellas una de las cuestiones más importantes es evadir la violencia y la pobreza secundaria a la que a veces son sometidas en los hogares encabezados por hombres.

No obstante, el interés de este artículo se centra en el ingreso debido a que se considera que éste debe de ser un criterio primordial para entender la pobreza, y permite separarla de otras privaciones que también pueden afectar a las mujeres pero que pueden responder a otras causas. Tal y como afirman Medeiros y Costa (2010: 95), la desigualdad de género se extiende más allá de la pobreza, y la pobreza no es exclusiva de las mujeres. Un mal diagnóstico acerca de estos hechos puede tener como consecuencia políticas equivocadas con resultados inadecuados en ambas áreas.

La razón para retener el concepto de ingreso como central tiene que ver, en primer lugar, con la definición de pobreza asumida en esta investigación. La pobreza es conceptualizada como la no participación en la sociedad debido a falta de recursos (Townsend, 1979; Nolan y Whelan, 1996; Rodríguez, 2010). De este modo, las privaciones multidimensionales que la pobreza representa siempre aparecen relacionadas a recursos insuficientes. Y esto diferencia el concepto de pobreza de otros más amplios, tales como el de exclusión social, que denota una falta de participación en la sociedad que puede ser causada por otros factores, como la discriminación, la discapacidad, las barreras territoriales, etc. (Burchardt et al., 2002). En el caso de la pobreza femenina la justificación radica en que independientemente de las condiciones de vida que una mujer pueda gozar en un momento determinado en el tiempo, su vulnerabilidad a la pobreza es muy alta si ella no tiene control sobre los recursos y carece de maneras independientes de sostenerse económicamente (Lister, 2010). 
Otra razón por la que se retiene el concepto de recursos es porque un predominio de los aspectos subjetivos conlleva el riesgo de distraerse de la fuente primordial de satisfacción de necesidades en la sociedad contemporánea: el ingreso. La falta de ingreso de muchas mujeres se debe a diferentes procesos por los cuales es discriminada en la sociedad. Ellos pueden ir desde salarios inferiores o dependencia económica de los hombres, hasta falta de acceso a la seguridad social o carencia de apoyos económicos por su condición de madres, etc. Desentrañar estos procesos es parte del interés de este artículo.

Particularmente en el caso de México, el ingreso ha sido un factor crítico para la pobreza. Mientras la cobertura de servicios públicos y de infraestructura ha mejorado sustancialmente en el país, la pobreza por ingreso se ha mantenido estancada (Boltvinik, 2003; Coneval, 2008). Por tanto, contrario a toda lógica, el hecho de que la población goce de mejores condiciones de salud y de mayor educación no ha significado un mejoramiento persistente en sus niveles de ingreso. La pobreza por ingresos se ha mantenido, con pequeñas oscilaciones, cercana a la mitad de la población, al menos desde principios de la década de los noventa (Boltvinik, 2003; Rodríguez, 2010 y 2011).

También es importante resaltar que la mayoría de las fuentes de información existentes permiten dar cuenta generalmente de los indicadores económicos y de otros aspectos mesurables. Por eso resulta importante saber cuánta información sobre la pobreza femenina en México podemos extraer de los indicadores existentes. No obstante, esta perspectiva va a ser complementada con la valiosa información que brindan algunos de los estudios etnográficos que hasta ahora se han realizado.

Existen tres instituciones sociales que median el acceso al ingreso y por tanto el riesgo de caer en la pobreza: el mercado laboral, el Estado y los hogares (Razavi y Staab, 2010). La interacción de las tres determina el estatus económico de las mujeres a través de sus vidas y distingue las causas de la pobreza femenina de las causas de la pobreza masculina. En particular, las causas de la pobreza femenina se encuentran tanto en la esfera doméstica privada de la familia, como en la esfera pública del mercado de trabajo y en el Estado de bienestar (Lister, 2010: 167-168).

Razavi y Staab (2010: 427) explican este proceso. Las instituciones que rigen el mercado de trabajo son la principal fuente que determina el acceso al ingreso así como la estratificación del trabajo en términos de clase y género. Las estrategias de desarrollo económico en los países 
pueden diferir en la medida en que son capaces de estimular el empleo de calidad para hombres y mujeres. Las mujeres enfrentan limitaciones adicionales tanto en la posibilidad de participar en el propio mercado como en la de recibir un salario decente, lo cual tiene que ver con la existencia de normas y prácticas sociales restrictivas. Por ejemplo, de acuerdo con Perrons (2010: 411) las mujeres se concentran en un rango de ocupaciones mal remuneradas, típicamente aquellas asociadas con labores de cuidado o de reproducción social tales como maestras, enfermeras, servidoras domésticas; también se encuentra que cuando las mujeres no trabajan en estas ocupaciones, ocupan posiciones bajas en la jerarquía ocupacional. También de acuerdo con Razavi y Staab (2010: 427), otra de las limitaciones adicionales radica en el hecho de que las mujeres cargan con la responsabilidad social de proveer cuidado a otros miembros del hogar, lo cual acarrea como consecuencia que suelan desempeñar trabajos más casuales donde reciben menores salarios y es limitado su acceso a la seguridad social. En otros casos, las labores de cuidado llegan incluso a privar totalmente a la mujer de participar en el mercado laboral. En resumen, los factores que generan desigualdad en el mercado laboral tienen que ver, en general, con una menor participación laboral de la mujer respecto a los hombres, con mayores tasas de informalidad y desempleo para las mujeres respecto a los hombres y con que, en igualdad de condiciones de nivel educacional y empleo, las mujeres pueden recibir un salario inferior al masculino (Costa y Silva, 2010).

La segunda institución que puede influir en el acceso al ingreso es la política social desarrollada por el Estado. De acuerdo con Marshall (1950), el principal mecanismo que compite con el mercado al generar oportunidades es el estatus de ciudadanía, especialmente en su componente social. Éste comprende el derecho a la obtención de los recursos y el acceso a los servicios (tales como la salud, la educación y los servicios sociales) que van a permitir a los ciudadanos ejercer el resto de sus derechos y cumplir con sus obligaciones. En este caso, el Estado puede proveer recursos mediante transferencias derivadas de los ingresos fiscales. También pueden usarse los recursos públicos para brindar servicios de cuidado. Es un importante mecanismo de redistribución de recursos en una sociedad porque tiene el potencial para mitigar la pobreza femenina creada por los mercados de trabajo (Razavi y Staab, 2010: 428).

En tercer lugar se encuentra el ámbito del hogar que puede influir en dos sentidos: en primer lugar, son las familias las que llevan a cabo 
las labores de reproducción de la fuerza de trabajo, algo que resulta esencial para la sociedad y el mercado, y son las mujeres las que asumen una parte desproporcionada de este trabajo; segundo, porque el acceso al ingreso que ganan los individuos aparece mediado por las relaciones dentro del hogar. Por tanto, la posibilidad de estar en pobreza depende del estatus económico de la persona, pero también del estatus económico de los otros miembros del hogar. En términos de género, la influencia del hogar en los recursos puede actuar en dos sentidos: por un lado, las mujeres podrían ser pobres aunque vivan en hogares no pobres debido a una desigual distribución de los recursos, y por otro, podrían escapar de la pobreza aunque no contaran con sus propios recursos si los ingresos se compartieran dentro del hogar. Sin embargo, si la mujer no cuenta con el derecho a transferencias gubernamentales que le provean de un respaldo económico, está en una situación de dependencia de los proveedores masculinos, disminuyendo su capacidad de decisión en asuntos domésticos y limitando sus posibilidades de abandonar el hogar en caso de que así lo desee (Razavi y Staab, 2010: 428).

Para explicar las condiciones concretas de la pobreza femenina en un contexto específico es importante desentrañar el peso que tiene la interrelación de estas tres instituciones. Ello puede ser observado de acuerdo con la sugerencia de Nauckhoff (2004: 50): no tratar al género como los hombres y las mujeres en general. Resulta fundamental establecer diferencias en términos de edad, grupos socioeconómicos y grupos étnicos, porque la situación de todos los grupos no es la misma. A esto se abocará este artículo.

\section{La feminización de la pobreza en México vista desde el modelo unitario y del modelo colectivo}

Antes de mostrar la evidencia que encontramos respecto a la pobreza femenina en México, resulta pertinente analizar brevemente los hallazgos de algunos estudios anteriores sobre este tema, con vistas a situar nuestro problema. Desde el análisis convencional de la pobreza femenina, mediante el modelo unitario, la evidencia cuantitativa coincide en afirmar que no existe feminización de la pobreza. En primer lugar, se encuentra que la tasa de pobreza femenina no es mayor que la tasa de pobreza masculina y que la situación de las mujeres en el tiempo, en vez de empeorar, ha mejorado sustancialmente (Damián, 2003; Rodríguez, 2012). También se encuentra que los hogares encabezados 
por mujeres son menos pobres que los encabezados por hombres (Sedesol, 2002; Damián, 2008; Villareal y Shin, 2008).

Sin embargo, como se mencionó anteriormente, una de las principales críticas a los estudios sobre pobreza femenina en general ha radicado en que se asume al hogar como un núcleo donde todos sus miembros son iguales y por tanto gozan de la misma cantidad de recursos, independientemente de quién los perciba. ${ }^{4}$ De este modo, si un hogar es pobre, todos sus miembros lo serán, y si un hogar es no pobre ninguno de sus miembros lo será. El modelo asume que todos los miembros del hogar son altruistas, ya que se comprometen asignando todo su esfuerzo monetario a maximizar la función de bienestar familiar; se ignora que los diferentes miembros del hogar puedan tener diferentes preferencias. Sin embargo, muchos estudios han mostrado que el último escenario es el más común en los hogares. Por tanto, el modelo unitario no deja apreciar las diferencias de género e intergeneracionales que existen en el interior de los hogares.

Ante lo anterior, y asumiendo que el reconocimiento de las desigualdades en el interior de los hogares es fundamental para entender la pobreza femenina (Sen, 2010), varios autores (Quisumbing, 2010; Falkingham y Baschieri, 2009, 2010) han propuesto la aplicación del modelo colectivo. Este modelo asume que las decisiones en el interior del hogar son el resultado de un proceso de negociación entre sus miembros. Los miembros que perciben ingreso escogen poner en la bolsa común algunos de sus recursos, mientras retienen otros para su consumo personal. Lo que determina el bienestar individual dentro de este modelo es la relativa fuerza de los hombres y de las mujeres en el proceso de negociación.

Por ello, la situación de los miembros que no ganan ingresos es diferente de la de aquellos que sí los reciben. El ingreso final de cada miembro del hogar será igual al ingreso equivalente de la bolsa común del hogar más lo que retenga de sus propios ingresos. Si un miembro del hogar no percibe ingresos, su ingreso final será solamente el ingreso equivalente de la bolsa común del hogar.

Si se considera que en el modelo unitario el bienestar de los miembros del hogar (Bhogar) se define por la siguiente fórmula, ${ }^{5}$ donde $I n g H$

${ }^{4}$ Cabe reconocer que esto en parte es consecuencia de la carencia de fuentes de información apropiadas, y no necesariamente de que otros autores desconozcan la importancia de tomar en cuenta las diferencias en el acceso a los recursos entre los miembros del hogar.

${ }^{5}$ Se asume que los menores no cuentan con fuentes de ingreso propias. 
es el ingreso masculino, Ing $M$ es el ingreso femenino, $H$ es hombres, $M$ es mujeres y $N$ es niños (Falkingham y Baschieri, 2009):

$$
\text { Bhogar }=(\operatorname{Ing} H+\operatorname{Ing} M) /(H+M+N)
$$

lo cual revela que dicho bienestar difiere al aplicar el modelo colectivo. Si $B H$ es el bienestar de los hombres, $B M$ el bienestar de las mujeres, $B N$ el bienestar de los niños, IngretH el ingreso retenido por los hombres, Ingret $M$ el ingreso retenido por las mujeres, IngcompH el ingreso de los hombres puesto en la bolsa común e IngcompM el ingreso compartido de las mujeres, entonces el bienestar de los diferentes miembros del hogar puede definirse como sigue (Falkingham y Baschieri, 2009):

$$
\begin{aligned}
& B H=\text { Ingret } H+[(\text { IngcompH+IngcompM }) /(H+M+N)] \\
& B M=\text { Ingret } M+[(\text { IngcompH+IngcompM }) /(H+M+N)]
\end{aligned}
$$

A su vez el bienestar de los niños será igual solamente al ingreso compartido dividido entre los distintos miembros (Falkingham y Baschieri, 2009): ${ }^{6}$

$$
B N=(\operatorname{IngcompH}+\operatorname{IngcompM}) /(H+M+N)^{7}
$$

En consecuencia, al aplicar este modelo puede encontrarse que no todas las personas en un hogar pobre son igual de pobres, y algunos pueden incluso no serlo. En un hogar no pobre también puede encontrarse que algunos miembros son pobres, mientras otros no lo son. La ventaja de aplicar este enfoque, para el caso que nos ocupa, es que permite centrar la atención en las mujeres pobres, sin importar el tipo de hogares en que vivan.

Obviamente los niveles de pobreza de los miembros del hogar bajo el modelo colectivo van a depender fuertemente de la asunción que

${ }^{6}$ Como parte del modelo colectivo existe otra asunción relacionada con el hecho de que las mujeres suelen compartir una mayor parte de sus recursos con los hijos (Falkingham y Baschieri, 2009). Aquí no se tomará en cuenta porque el interés primordial de este trabajo se centra en las características de la pobreza femenina (no la infantil), se asume la variación más ligera del modelo unitario. Ello con el propósito de examinar cómo un pequeño cambio afecta las tasas de pobreza masculina y femenina.

7 Aunque se explica la racionalidad del modelo colectivo para el bienestar de los menores, esta parte del modelo no se incluye en el artículo porque no es el interés de este documento referirse a la pobreza infantil. 
se realice sobre el porcentaje de retención de recursos por parte de aquellos miembros que ganan ingresos. En el caso de México no existe ninguna fuente que presente información fidedigna sobre este tema.

Rodríguez (2012) aplicó el modelo colectivo para analizar la pobreza femenina en México usando la Línea de Bienestar de Coneval. La principal conclusión de su estudio, como se mencionó anteriormente, es que desde ese modelo tampoco puede hablarse de que exista feminización de la pobreza en México, ${ }^{8}$ pero sí se encuentran tasas de pobreza femenina mayores a las masculinas. El cuadro 1 presenta estos resultados usando la retención más pequeña de todas las que se muestran. Se trata de la asunción de que tanto hombres como mujeres que ganan ingresos retienen para sí el 20\% de los mismos, y ponen el resto en la bolsa común del hogar. Esto es comparado con la pobreza de hombres y mujeres aplicando el modelo unitario convencional.

Como puede apreciarse, al aplicar el modelo colectivo la tasa de pobreza de las mujeres se incrementa ligeramente, pero la de los hombres baja sustancialmente, de modo que la distancia entre unas y otras se hace muy grande. El cuadro 2 muestra cómo estas tasas se distribuyen entre los hogares encabezados por hombres y los encabezados por mujeres.

Las tasas de pobreza femenina son más altas en los hogares encabezados por hombres. Al aplicar el modelo colectivo la tasa femenina se incrementa en este tipo de hogares a la vez que la masculina baja de manera importante, generando una distancia muy fuerte entre la situación de los hombres y la de las mujeres. Ambos hallazgos coinciden con estudios cualitativos tales como los de García y Oliveira (1994), Chant (1998) y González de la Rocha (1994), quienes afirman que la pobreza femenina en México es mayor, pero que se encuentra fundamentalmente en los hogares encabezados por hombres.

\section{Sobre el cálculo del ingreso y la línea de pobreza}

En este trabajo se usa la misma metodología seguida en Rodríguez (2012) para el cálculo de las características de la pobreza femenina

8 Ello debido a que no se cumplen dos de las tres condiciones consideradas dentro de la noción de feminización de la pobreza (Chant, 2007): la situación de las mujeres no ha empeorado con el tiempo, ni los hogares encabezados por mujeres son más pobres que los hogares encabezados por hombres. 
CUADRO 1

Tasa de pobreza de mujeres y hombres usando el modelo unitario y el colectivo con la Línea de Bienestar de Coneval (2008)

\begin{tabular}{lcc}
\hline Modelo & Mujeres & Hombres \\
\hline Modelo unitario & 44 & 42 \\
$\begin{array}{l}\text { Modelo colectivo asumiendo una retención del 20\% } \\
\text { para hombres y mujeres }\end{array}$ & 47 & 34 \\
\hline
\end{tabular}

FUENTE: Rodríguez, 2012.

bajo el modelo colectivo. Se aplica el modelo colectivo de hogar de la misma manera en que fue mostrado en el apartado anterior; es decir, asumiendo que tanto los hombres como las mujeres que perciben ingresos retienen para sí $20 \%$ de los mismos. Ante la falta información precisa sobre cómo son distribuidos los recursos en el interior de los hogares, se trata de una asunción arbitraria, no obstante se usa ésta con el propósito de ver cómo una ligera variación del modelo unitario varía la pobreza femenina.

Para calcular el ingreso con objeto de medir la pobreza se siguieron varios pasos. Primero se le asignó a cada persona todo el ingreso que se encuentra clasificado por persona en la ENIGH; esto es, ingreso por trabajo, negocios, cooperativas, renta de capital, transferencias y pago en especie. La asignación se hizo para todos los perceptores del hogar independientemente de que estuvieran ocupados o no. Es importante aclarar que en el caso de los regalos provenientes de otros hogares y las transferencias en especie en algunas ocasiones aparecen atribuidos

\section{CUADRO 2}

Tasa de pobreza de mujeres y hombres usando el modelo unitario y el colectivo según el género del jefe de hogar con la Línea de Bienestar de Coneval (2008)

\begin{tabular}{|c|c|c|c|c|}
\hline \multirow[b]{2}{*}{ Modelo } & \multicolumn{2}{|c|}{$\begin{array}{c}\text { Hogares encabezados } \\
\text { por hombres }\end{array}$} & \multicolumn{2}{|c|}{$\begin{array}{l}\text { Hogares encabezados } \\
\text { por mujeres }\end{array}$} \\
\hline & Mujeres & Hombres & Mujeres & Hombres \\
\hline Modelo unitario & 46 & 43 & 41 & 39 \\
\hline $\begin{array}{l}\text { Modelo colectivo asumiendo } \\
\text { una retención del } 20 \% \text { para hombres } \\
\text { y mujeres }\end{array}$ & 51 & 34 & 40 & 34 \\
\hline
\end{tabular}

FUENTE: Rodríguez, 2012. 
a personas específicas y en otros al hogar en su conjunto. Solamente cuando se cumplió el primero de los dos casos fue que este tipo de ingreso fue asignado a personas específicas. Si no, fue reservado, junto con el ingreso por autoconsumo (que en ningún caso aparece atribuido por persona), como ingreso que formó parte de la bolsa común del hogar.

El segundo paso consistió en sustraer de los ingresos personales el porcentaje que se consideró retenido (en este caso 20\%), y dejarlo como ingreso exclusivo de cada persona. El ingreso restante (es decir, $80 \%$ del ingreso personal), junto con el ingreso reservado mencionado anteriormente se suma a la bolsa común del hogar.

El tercer paso consiste en aplicar a la bolsa común del hogar la escala de equivalencia utilizada. De este modo el ingreso final de cada miembro del hogar será igual al ingreso equivalente del hogar más el que ya se había considerado como ingreso exclusivo de ese miembro. Si el miembro no percibe ingreso, su ingreso final será solamente igual al ingreso equivalente del hogar. De este modo los ingresos finales de los distintos miembros del hogar pueden ser totalmente diferentes. Es ese ingreso final el que resulta comparable con la línea de pobreza.

Como criterio de pobreza se usa la Línea de Bienestar económico de Coneval. Ello porque de acuerdo con este organismo, es la línea que identifica a la población que no cuenta con los recursos suficientes para adquirir los bienes y servicios que requiere para satisfacer sus necesidades alimentarias y no alimentarias. No se usa la Línea de Bienestar Mínimo porque ésta sólo se refiere a aquella población que incluso haciendo uso de todo su ingreso no puede satisfacer sus necesidades alimentarias (Coneval, 2009), lo que no resultaría de ninguna utilidad para este estudio. Por otro lado, es importante aclarar que si bien la Línea de Bienestar no constituye exactamente la medida oficial, sino solamente un componente de la misma, resulta más apropiada para los fines de este trabajo, pues lo que se intenta es medir la pobreza de las mujeres en términos de los ingresos que realmente tiene a su disposición, y no caracterizar la situación de la mujer de acuerdo con la situación general del hogar, que sería lo que se estaría midiendo si se usara la Medida de Pobreza Multidimensional. ${ }^{9}$

${ }^{9}$ La Medida de Pobreza Multidimensional de Coneval considera que las personas son pobres sólo si cumplen los dos requisitos: ser pobres por ingreso (es decir, estar debajo de la Línea de Bienestar Económico), y ser pobres por carencias sociales (es decir, tener al menos una carencia social). Las carencias sociales dependen más de la situación general del hogar que de condiciones individuales. 
Para construir el ingreso que va a ser comparado con la línea de pobreza se siguen los mismos criterios de Coneval. Se usa solamente el ingreso corriente (remuneraciones al trabajo, ingreso por negocios, renta de capital, transferencias, ingreso por cooperativas, valor imputado por autoconsumo, valor imputado al pago en especie y regalos en especie que se reciben más de una vez al año). Se eliminan del ingreso usado para medir la pobreza los regalos en especie no recibidos más de una vez al año y la estimación del alquiler de la vivienda. Las escalas de equivalencia aplicadas a la bolsa común del hogar son igualmente las usadas por Coneval: 0.70 para personas de 0 a 5 años; 0.74 para personas de 6 a 12 años; 0.71 para personas de 13 a 18 años y 0.99 para personas mayores de 19 años.

Hasta aquí nos hemos referido a la Línea de Bienestar de Coneval en términos oficiales. Sin embargo esta línea está sujeta a muchos cuestionamientos por la manera en que calcula la pobreza (Boltvinik, 2009; Rodríguez, 2011). La Línea de Bienestar se basó primeramente en el uso de una canasta alimentaria construida por expertos (esta fue denominada Línea de Bienestar Mínimo). Sin embargo para costear la canasta alimentaria se escogió a un grupo de referencia que cubre el "costo energético definido al costo más eficiente posible" (Coneval, 2009: 71), lo que hace que se dejen fuera otras consideraciones tales como el tipo de nutrientes que se estaban incluyendo. De este modo, el grupo escogido como referencia es muy pobre debido a que los hogares pobres suelen tener un mayor consumo calórico porque escogen alimentos altos en energía pero bajos en nutrientes. Este tipo de alimentos resultan más eficientes para proporcionarles la energía necesaria para realizar sus actividades diarias (por ejemplo, es común encontrar que los hogares pobres prefieran consumir refrescos altamente calóricos antes que leche). Entonces los gastos que se tomaron como base para determinar la Línea de Bienestar Mínima son los gastos de hogares pobres. Asimismo, para incluir la canasta básica no alimentaria (que es el segundo componente de la Línea de Bienestar) fue usado este estrato poblacional de referencia. El coeficiente de Engel se calculó usando los gastos no alimentarios de este estrato. Se obtuvo la Línea de Bienestar al dividir el costo de la canasta alimentaria entre el recíproco del coeficiente de Engel. Después de que se tenía establecido el costo de la Línea de Bienestar se determinó qué rubros iban a ser incluidos como parte de la canasta básica no alimentaria y su valor total se repartió entre éstos. Con este procedimiento se obtuvo una línea de pobreza muy baja que representa el gasto de los hogares 
muy pobres. Por ejemplo, en el rubro de vivienda y servicios de conservación para áreas urbanas se contempla un costo de 141.52 pesos por persona (Coneval, 2009). Ello implica que si una persona vive sola, con eso tiene que pagar la renta mensual y los servicios de la vivienda. Como cualquier persona que esté familiarizada con los costos de la vivienda urbana sabe, esto resulta imposible. La razón por la que salió un costo tan bajo es porque el estrato de referencia debe de vivir en asentamientos irregulares o viviendas precarias donde no se paga renta y se pagan muy pocos servicios o ninguno. De este modo, quedó una línea de pobreza cuyo valor total es igual al doble de la canasta alimentaria mínima, por lo que se asume que en México se puede vivir y no ser pobre con esta cantidad de dinero. Todos estos gastos están calculados por persona, sin embargo, en los hogares con más de una persona se usó un procedimiento que resulta sumamente cuestionable porque contribuye a bajar aún más el valor de la línea de pobreza. Se usaron escalas de equivalencia que hacen que para las personas menores de 18 años requieran en promedio sólo $72 \%$ de esta línea. En los hogares efectivamente existen economías de escala, por lo que la manera más adecuada de calcular la pobreza es usando escalas de equivalencia. Sin embargo en este caso no es procedente porque se trata de líneas muy bajas construidas de una manera muy estricta por persona. El resultado es que la forma en que está expresado el ingreso y la forma en que está expresada la línea de pobreza no son comparables en términos matemáticos. ${ }^{10}$

También para los efectos de esta investigación hay un elemento que es muy importante tomar en consideración. Como el centro de este trabajo es caracterizar la pobreza femenina desde el modelo colectivo, y dicho modelo asume una retención de un porcentaje del ingreso por aquellos que lo reciben, es sumamente importante tomar en cuenta los costos adicionales que tiene generar este ingreso. Ello porque aquellas personas que trabajan fuera del hogar requieren en muchos casos consumir alimentos fuera y realizar gastos en el transporte diario. Lo mismo se aplicaría para el caso de los estudiantes de niveles de educación media superior, superior y posgrado, porque igualmente incurren en estos gastos como parte de sus actividades cotidianas. ${ }^{11}$ Ante esta situación se construyó una línea de pobreza

${ }^{10}$ Se agradecen los comentarios de los dictaminadores anónimos sobre este tema.

${ }^{11}$ Igualmente se agradecen los comentarios de los dictaminadores anónimos, pues resulta esencial tomar en consideración los costos adicionales que tiene generar el ingreso si se asume el modelo colectivo de hogar. 
alternativa a la Línea de Bienestar de Coneval que incluye un valor más alto para quienes trabajan o estudian fuera del hogar.

El procedimiento para estimar estos costos adicionales fue el siguiente. Cabe mencionar que se trata simplemente de un estimado porque excede las posibilidades de esta investigación calcular el costo de una comida económica diaria fuera de casa a nivel nacional para 2008, pues sería necesario investigarlo en cada uno de los estados, así como en las áreas urbanas y rurales. Por tanto, el procedimiento de estimación utilizado se basó en los hallazgos de Evalúa DF, que construyó y costeó una canasta normativa alimentaria por grupos de edad y sexo en el Distrito Federal en el año 2011, atendiendo a procedimientos que reconocen umbrales adecuados a las percepciones y a las prácticas de la población, así como al criterio de expertos (Evalúa DF, 2011). Basados en este ejercicio se encuentra que el costo de realizar una comida fuera del hogar por cinco días a la semana durante las cuatro semanas que tiene el mes es equivalente a agregar $40 \%$ más al valor de la canasta alimentaria de Coneval. ${ }^{12}$ Ello se determinó usando aquel grupo por edad y sexo cuya estimación del valor de la canasta alimentaria resulta igual que la de Coneval. ${ }^{13}$ Por tanto, para todas las personas que estudian o trabajan fuera del hogar se le agregó $40 \%$ más al valor de la Línea de Bienestar Mínimo tanto para las áreas urbanas como para las rurales.

Asimismo se estimó el costo del transporte público equivalente a dos viajes diarios (ida y vuelta) al trabajo o estudio. Para ello se usó un promedio de lo que costaba el viaje en 2008 en varias ciudades del país, ${ }^{14}$ que era de 5 pesos, por lo que se optó por 10 pesos diarios. En áreas rurales la propia ENIGH determinó que el costo de un viaje tam-

${ }^{12}$ El cálculo de Evalúa DF en el rubro de alimentos fuera del hogar incluye sólo el costo adicional, porque para su cálculo se restó del costo de consumir alimentos fuera del hogar el costo de adquirirlos para prepararlos en el hogar (Boltvinik, 2012).

${ }^{13}$ Se trata del grupo de mujeres de 51 a 70 años cuyo valor de la Canasta Normativa Alimentaria DF es 99\% del valor de la Canasta de Coneval para el 2011 (Evalúa DF, 2011).

${ }^{14}$ Para estimar la tarifa de un viaje en transporte público para 2008 se usó información de varias ciudades situadas en diferentes estados. Se les escogió porque tenían alguna información disponible en internet. Se encontró que en Guadalajara costaba 4.50 pesos <http:/ /www.informador.com.mx/jalisco/2009/163388/6/es-inminente-unaumento-a-la-tarifa-del-transporte-publico.htm>; en Nuevo León costaba entre 4.00 y 6.50 pesos <http://www.nl.gob.mx/?P=aet_comof_tarifas2008>; en el Distrito Federal costaba entre 3.00 y 5.00 pesos el microbús y 2.00 pesos el metro $<$ http://www.setravi. df.gob.mx/wb/stv/tarifas_autorizadas_tp $>$ y $<$ http://www.metro.df.gob.mx/organismo/ costoboleto.html>; en la ciudad de León, Guanajuato, costaba entre 6.00 y 8.00 pesos <http:/ / oruga-sit.leon.gob.mx/noticias.php?id=89> (consulta realizada el 28 de febrero). Se sacó un promedio de todo y se obtuvo que el viaje individual costaba 5 pesos. 
bién estaba cercano a esa cantidad, sobre todo porque muchos de los habitantes de las áreas rurales se trasladan a las áreas urbanas cercanas para trabajar o estudiar. El cálculo del transporte diario para ir a trabajar o estudiar fue de 200 pesos mensuales. Sin embargo la Línea de Bienestar estimaba ya una cantidad para transporte. Se consideró que para las áreas urbanas podían realizarse como promedio dos viajes a la semana (ida y vuelta) para asuntos personales y familiares (tales como compra de alimentos o visitas familiares u ocio). Por tanto, se restó de la cantidad ya considerada para transporte, el costo de los viajes para asuntos personales. La cantidad sobrante fue restada de los 200 pesos, con lo cual en estas áreas se agregaron 134 pesos al costo del transporte ya considerado. Para las áreas rurales se consideró que como promedio se realizaba un viaje semanal para asuntos familiares, ocio o abasto de alimentos, ya que en muchos casos debido a la cercanía estas actividades pueden realizarse sin necesidad de usar transporte público. En este caso se restó el equivalente a un viaje a la semana de la cantidad ya considerada para transporte. El sobrante se restó de los 200 pesos, con lo cual fue necesario sumar 155 pesos.

En total, el valor de la Línea de Bienestar se incrementó 25\% para las áreas urbanas y $33 \%$ para las áreas rurales por concepto de alimentos fuera del hogar y transporte de todas aquellas personas que estudian (de nivel bachillerato hacia arriba) o trabajan fuera del hogar. Asimismo, con la nueva línea calculada se intenta corregir el procedimiento usado por Coneval de calcular los gastos por persona y luego aplicar escalas de equivalencia. En este caso se omitió la aplicación de las escalas de equivalencia. El ingreso para comparar con la línea de pobreza se calculó por persona. De este modo el ingreso del hogar y la línea de pobreza se expresan en los mismos términos matemáticos. Con las modificaciones descritas en la Línea de Bienestar de Coneval se calcularon nuevamente la pobreza femenina y la masculina.

El cuadro 3 muestra la tasa de pobreza femenina y la masculina usando el modelo colectivo de hogar con la retención del 20\%, tanto bajo la Línea de Bienestar de Coneval como bajo la Línea de Bienestar de Coneval Modificada. ${ }^{15}$ Si bien al usar una u otra se obtienen diferentes tasas de pobreza para los hombres y para las mujeres, es claro que si se usa el modelo colectivo de hogar en ambas líneas, la pobreza

${ }_{15}$ En resumen la Línea de Bienestar de Coneval Modificada toma en cuenta los costos de transporte y alimentos fuera del hogar para aquellas personas que trabajan o que estudian bachillerato o niveles superiores. Además no aplica escalas de equivalencia sino que el ingreso que se compara con la línea de pobreza está calculado por persona. 
femenina es sustancialmente mayor que la masculina. Conforme a la línea original de Coneval la tasa de pobreza femenina es de $47 \%$ y la brecha respecto a la pobreza masculina es de 13\%. Bajo la Línea de Coneval Modificada la tasa de pobreza femenina es mucho mayor $(57 \%)$, pero la brecha de pobreza con el género masculino resulta un poco menor $(9 \%)$. Estos últimos resultados son lógicos por el hecho de que la Línea de Bienestar Modificada es más elevada que la Línea de Bienestar, lo que automáticamente hace que se incremente la pobreza en ambos géneros.

A la luz de estos datos puede considerarse un resultado contundente el hecho de que si se aplica el modelo colectivo de hogar, la pobreza femenina en México resulta superior a la masculina. Ello se verifica incluso asumiendo una retención del ingreso personal baja y tomando en cuenta los costos de producir el ingreso. Como hipótesis podemos plantear que usando los criterios definidos oficialmente $u$ otros muy cercanos a éstos, ${ }^{16}$ la pobreza femenina oscila entre un 47 y un $57 \%$ de las mujeres, mientras que la pobreza masculina oscila entre un 34 y un $48 \%$ de los hombres. Claramente se trata de una hipótesis sobre las brechas de género en la pobreza debido a que como vimos, la Línea de Bienestar de Coneval está sujeta a importantes críticas, y la Línea de Bienestar Modificada propuesta tampoco puede considerarse exacta porque es un ejercicio que utiliza supuestos que no son enteramente reales debido a la dificultad para calcular con exactitud el costo del transporte y la alimentación fuera del hogar en el ámbito nacional para el año 2008. Al construir la segunda línea se perseguía simplemente conocer los resultados de la pobreza femenina con el modelo colectivo de hogar tomando en cuenta los costos de producir ingreso. Pero lo que sí puede afirmarse es que la pobreza femenina se mueve entre un rango y otro y es claramente superior a la masculina. En cualquier caso se trata de un estimado, como también lo es la cantidad de recursos que se asume que retienen los miembros del hogar en este ejercicio.

No obstante, se optó por mantener los resultados bajo la línea original de Coneval debido a que como se explicó anteriormente, la línea modificada no constituye una línea que pueda sostenerse de manera exacta dado que está basada en supuestos y extrapolaciones

16 Aun cuando se use la Línea de Bienestar de Coneval Modificada, los criterios que se utilizan, aunque ligeramente superiores, siguen cercanos a los oficiales. Para conocer medidas de pobreza alternativas para el caso de México véase por ejemplo Boltvinik, 2001. 


\section{CUADRO 3}

Tasa de pobreza de mujeres y hombres bajo el modelo colectivo con la Línea de Bienestar de Coneval y la Línea de Bienestar de Coneval Modificada (2008)

\begin{tabular}{lcc}
\hline Modelo & Mujeres & Hombres \\
\hline $\begin{array}{l}\text { Línea de Bienestar de Coneval } \\
\text { (retención del 20\% del ingreso) }\end{array}$ & 47 & 34 \\
$\begin{array}{l}\text { Línea de Bienestar de Coneval modificada } \\
\text { (retención del 20\% del ingreso) }\end{array}$ & 57 & 48 \\
\hline
\end{tabular}

FUENTE: Elaboración propia con base en la Encuesta Nacional de Ingreso y Gasto de los Hogares 2008.

que en ningún caso presentan una información fiel respecto al costo de alimentos y transporte fuera del hogar para el 2008 a nivel nacional.

\section{La otra cara de la pobreza femenina: del modelo unitario al modelo colectivo}

Anteriormente se mostró cómo al usar el modelo colectivo se evidencia una brecha de género en la pobreza. Aunque claramente se trata de una aproximación, este modelo puede considerarse una asunción más realista que la del modelo unitario (Rodríguez, 2012). Ello debido a que como se expuso en la introducción, numerosos estudios documentan que el ingreso no se comparte en su totalidad dentro de la familia, y que son las mujeres las que suelen ser de las más perjudicadas en su bienestar.

En esta parte, el interés es examinar cómo el género se cruza con otras divisiones sociales tales como el lugar de residencia, la etnicidad, la edad, la educación y el estatus ocupacional para determinar mayores tasas de pobreza para las mujeres. En la sociedad existen distinciones muy importantes entre grupos de personas; estos grupos son socialmente sancionados como diferentes tanto en términos materiales como culturales. Las divisiones sociales persisten en la sociedad porque las reproducen las creencias culturales dominantes, la organización de las instituciones sociales y la interacción de los individuos. Pertenecer a alguna división social en específico confiere al individuo oportunidades desiguales de acceso a los recursos sociales, y por tanto oportunidades de vida y estilos de vida diferentes (Payne, 2006: 348). El género 
es una de las divisiones sociales más importantes en la sociedad, sin embargo, también es atravesado por otras divisiones, y en este proceso se crean diferentes oportunidades de vida. Por tanto, no debe hablarse del género en general debido a que la situación de todos los grupos sociales no es la misma. El objetivo es conocer aquellas variables que elevan la tasa de la pobreza femenina con vistas a establecer un primer orden de hallazgos que puedan resultar relevantes para la política.

Para ello se expondrán estadísticas descriptivas que muestran la relación de la pobreza con cada una de las variables por separado; asimismo se comparará la variación que puede llegar a existir en la brecha de género si se pasa del modelo unitario al colectivo. Las estadísticas calculadas con el factor de expansión de la ENIGH 2008 indican lo siguiente.

El lugar de residencia de las personas, ya sea en áreas urbanas o rurales, puede establecer diferencias importantes respecto a las tasas de pobreza debido a que las oportunidades de empleo y la calidad de los servicios que existen varían sustancialmente entre un ámbito y otro. En el cuadro 4 se muestran las diferencias entre las tasas de pobreza por lugar de residencia aplicando ambos modelos. Como puede apreciarse existe un cambio marcado en la brecha de pobreza entre hombres y mujeres al pasar del modelo unitario al colectivo. Encontramos que bajo el modelo unitario si bien las tasas son mucho mayores en el ámbito rural, las brechas entre hombres y mujeres en ambos ámbitos son mínimas. Bajo el modelo colectivo la brecha de pobreza entre hombres y mujeres aumenta sustancialmente y dicha brecha resulta también muy similar para ambas áreas (aproximadamente un $14 \%$ ), si bien las tasas siguen siendo muy superiores en el ámbito rural.

\section{CUADRO 4}

Tasa de pobreza de hombres y mujeres bajo el modelo unitario y colectivo, según lugar de residencia, usando la Línea de Bienestar de Coneval (2008)

\begin{tabular}{lccccc}
\hline & \multicolumn{2}{c}{ Modelo unitario } & & \multicolumn{2}{c}{ Modelo colectivo } \\
\cline { 2 - 3 } \cline { 5 - 6 } & Hombres & Mujeres & & Hombres & Mujeres \\
\hline Rural* & 59 & 60 & & 50 & 64 \\
Urbano & 38 & 40 & & 30 & 43 \\
\hline
\end{tabular}

* Localidades con menos de 2500 habitantes.

FUENTE: Elaboración propia con base en la Encuesta Nacional de Ingreso y Gasto de los Hogares 2008. 
Rodríguez, UNA CARACTERIZACIÓN DE LA POBREZA FEMENINA

\section{CUADRO 5}

Tasa de pobreza de hombres y mujeres bajo el modelo unitario y colectivo, según condición de etnicidad, usando la Línea de Bienestar de Coneval (2008)

\begin{tabular}{lcccccc}
\hline & \multicolumn{2}{c}{ Modelo unitario } & & \multicolumn{2}{c}{ Modelo colectivo } \\
\cline { 2 - 3 } \cline { 5 - 6 } & Hombres & Mujeres & & Hombres & Mujeres \\
\hline Perteneciente a grupo étnico* & 71 & 73 & & 58 & 70 \\
No perteneciente a grupo étnico & 40 & 42 & & 29 & 42 \\
\hline
\end{tabular}

* Se considera a la persona que declara hablar una lengua indígena.

FUENTE: Elaboración propia con base en la Encuesta Nacional de Ingreso y Gasto de los Hogares 2008.

El cuadro 5 muestra las diferencias entre tasas de pobreza por condición de etnicidad si se usan ambos modelos. Se encuentra que las tasas de pobreza tanto de hombres como de mujeres son sustancialmente más altas cuando pertenecen a grupos étnicos bajo ambos modelos. Con el modelo unitario la brecha entre hombres y mujeres es muy baja, tanto para los grupos étnicos como para el resto de la población. Al pasar al modelo colectivo puede apreciarse que las brechas entre hombres y mujeres se incrementan de un modo muy similar tanto para los grupos étnicos como para el resto de la población.

El cuadro 6 muestra una comparación similar a las anteriores, pero ahora por grupos de edad. En el caso de los menores de 18 años se observa que no hay un cambio en la brecha de género al pasar del modelo unitario al colectivo, aunque sí se incrementa su tasa. Este resultado se debe a que esta categoría mayormente no percibe ingresos, sino que depende del ingreso de los adultos. Un cambio de modelo no genera diferencias de género, pero sí incrementa su tasa, porque los adultos de los cuales depende retienen una proporción mayor del ingreso. En el caso de los jóvenes de 18 a 19 años se encuentra que un cambio de modelo aumenta la tasa de pobreza, pero es un aumento ligero para los hombres y más grande para las mujeres, con lo cual se encuentra una brecha de género en esta categoría de edad; ello se debe a que si bien la mayoría de los hombres todavía no perciben ingresos (por eso su tasa de pobreza no disminuye), los reciben en mayor medida que las mujeres de esta edad.

Sin embargo para el resto de las edades los hombres bajan su tasa de pobreza con el modelo colectivo. A medida que la edad va avanzando la tasa de pobreza de los hombres baja en mucha menor medida. 


\section{CUADRO 6}

Tasa de pobreza de hombres y mujeres bajo el modelo unitario y colectivo, según grupos de edad, usando la Línea de Bienestar de Coneval (2008)

\begin{tabular}{cccccc}
\hline & \multicolumn{2}{c}{ Modelo unitario } & & \multicolumn{2}{c}{ Modelo colectivo } \\
\cline { 2 - 3 } \cline { 5 - 6 } Menores 18 & Hombres & Mujeres & & Hombres & Mujeres \\
\hline $18-19$ & 57 & 58 & & 62 & 63 \\
$20-24$ & 42 & 47 & & 43 & 54 \\
$25-29$ & 38 & 45 & & 34 & 49 \\
$30-34$ & 40 & 46 & & 31 & 49 \\
$35-39$ & 46 & 50 & & 32 & 51 \\
$40-44$ & 46 & 47 & & 33 & 48 \\
$45-49$ & 43 & 42 & & 29 & 44 \\
$50-54$ & 37 & 37 & & 27 & 40 \\
$55-59$ & 37 & 38 & & 30 & 41 \\
$60-64$ & 39 & 39 & & 33 & 43 \\
$65-69$ & 41 & 44 & & 38 & 49 \\
$70-74$ & 47 & 50 & & 45 & 54 \\
$75-79$ & 49 & 47 & & 46 & 50 \\
80 y más & 51 & 48 & & 48 & 53 \\
& 55 & 46 & 54 & 51 \\
\hline
\end{tabular}

FUENTE: Elaboración propia con base en la Encuesta Nacional de Ingreso y Gasto de los Hogares 2008.

Y al final de la distribución de edad, después de los 80 años, casi no varía nada. Ello puede deberse a que los ingresos que se perciben van disminuyendo con la edad, con lo cual la cantidad de ingreso que los hombres pueden manejar en ese momento de su vida es mucho menor. También puede deberse a que los hombres que viven hasta edades avanzadas tienen menos probabilidades de que esté viva su pareja, con lo cual comparten menos sus recursos y por tanto no se encuentra una gran diferencia en su situación al cambiar de un modelo a otro.

Las mujeres bajo el modelo unitario tienen tasas de pobreza más o menos similares a las de los hombres, con pequeñas diferencias. $\mathrm{Al}$ pasar al modelo colectivo encontramos que la tasa de pobreza de las mujeres se incrementa para todas las edades, y que la brecha de pobreza entre hombres y mujeres es mucho mayor. Sin embargo el com- 
portamiento de la brecha no es uniforme, sino que presenta la forma de una $\mathrm{U}$ invertida, ya que se incrementa sostenidamente hasta los 30-34 años y a partir de ahí comienza a descender para ser mucho menor en las edades mayores. Este resultado puede deberse a que las mujeres son más pobres durante el periodo reproductivo porque al asumir la responsabilidad por el cuidado de sus hijos, su participación en el mercado laboral disminuye. A medida que se alejan de la edad reproductiva su participación en el mercado laboral es mayor. En la tercera edad la brecha continúa disminuyendo, presumiblemente porque muchas mujeres ya no tienen viva a su pareja, con lo cual disponen de todo el ingreso familiar para ellas, ya sea que éste proceda en forma de pensión como resultado de su propia participación en el mercado laboral, o de que cuenten con la pensión por viudez, o que reciban ayuda de familiares que viven fuera del hogar.

También al analizar la pobreza femenina en relación con la edad encontramos que las mujeres adultas entre los 20 y los 39 años tienen en promedio tasas de pobreza ligeramente inferiores a las de su contraparte en la tercera edad (es decir, las mujeres de 60 a 79 años) bajo ambos modelos, con lo cual no puede afirmarse que la pobreza femenina esté atravesando por un proceso dinámico de empeoramiento en el tiempo.

En el cuadro 7 se analiza cómo varía la brecha de pobreza entre hombres y mujeres según su nivel de educación al pasar del modelo unitario al modelo colectivo, y los resultados son interesantes. Se encuentra, sin sorpresa, que la pobreza baja notablemente a medida que aumenta el nivel educacional tanto para los hombres como para las mujeres bajo ambos modelos. También se encuentra que para todas las categorías educacionales la tasa de pobreza de los hombres baja cuando se pasa del modelo unitario al modelo colectivo. Para las mujeres encontramos que si bien bajo el modelo unitario tienen tasas de pobreza muy similares a las de los hombres según el nivel educacional, al pasar al modelo colectivo la situación varía. En los primeros niveles las mujeres aumentan su tasa de pobreza, es decir, hasta la preparatoria completa. Por encima de ahí se quedan con las mismas tasas de pobreza que bajo el modelo unitario. Ello implica que existe una influencia determinante entre una mayor educación y una menor pobreza. A pesar de que estas últimas tasas de pobreza se quedan sin cambio, la brecha de pobreza entre los hombres y las mujeres aumenta para todos los niveles educacionales al pasar del modelo unitario al modelo colectivo. Ello debido a que los salarios de los hombres siempre 
CUADRO 7

Tasa de pobreza de hombres y mujeres bajo el modelo unitario y colectivo, según nivel educacional, usando la Línea de Bienestar de Coneval (2008)

\begin{tabular}{lccccc}
\hline & \multicolumn{2}{c}{ Modelo unitario } & & \multicolumn{2}{c}{ Modelo colectivo } \\
\cline { 2 - 3 } \cline { 5 - 6 } \cline { 5 - 6 } Sin educación & Hombres & Mujeres & & Hombres & Mujeres \\
Alfabetos & 68 & 69 & & 63 & 73 \\
Primaria completa & 51 & 59 & & 53 & 63 \\
Secundaria completa & 42 & 52 & & 40 & 56 \\
Preparatoria completa & 28 & 44 & & 30 & 46 \\
Educación técnica & 19 & 27 & & 19 & 29 \\
Licenciatura & 9 & & & 10 & 21 \\
Posgrado & 4 & 2 & & 3 & 10 \\
\hline
\end{tabular}

FUENTE: Elaboración propia con base en la Encuesta Nacional de Ingreso y Gasto de los Hogares 2008.

son mayores que los de las mujeres para niveles de escolaridad similares (CEPAL, 2008: tabla 1.7.1). Presumiblemente esto se debe a que con igual nivel de escolaridad, los empleadores prefieran a los hombres para ocupar puestos de mayor categoría, con lo cual ellas desempeñan puestos menos remunerados. De hecho, los ingresos femeninos de las trabajadoras de todos los niveles educacionales representan en promedio $80 \%$ de los ingresos masculinos (CEPAL, 2008: tabla 1.7.1). Por tanto, cuando los hombres comparten una menor parte de su ingreso, su situación de pobreza mejora.

El estatus ocupacional de las personas resulta muy importante para determinar su situación de pobreza. En el cuadro 8 se muestra la relación entre el género y el estatus ocupacional en ambos modelos. Se encuentra que la tasa de pobreza de los hombres al pasar del modelo unitario al colectivo sólo disminuye notablemente cuando se trata de hombres ocupados, pero en el caso de aquellas dos categorías que no perciben ingreso, tales como los desempleados y los inactivos, las tasas de pobreza aumentan al pasar al modelo colectivo. Por otro lado, se encuentra que las mujeres no siempre aumentan sus tasas de pobreza al pasar del modelo unitario al colectivo; por el contrario, las mujeres empleadas disminuyen sus tasas de pobreza, y bajo el modelo colectivo en esta categoría su tasa es similar a la de los hombres, de ahí que no 
CUADRO 8

Tasa de pobreza de hombres y mujeres bajo el modelo unitario y colectivo, según estatus ocupacional, usando la Línea de Bienestar de Coneval (2008)

\begin{tabular}{lccccc}
\hline & \multicolumn{2}{c}{ Modelo unitario } & & \multicolumn{2}{c}{ Modelo colectivo } \\
\cline { 2 - 3 } \cline { 5 - 6 } \cline { 5 - 6 } & Hombres & Mujeres & & Hombres & Mujeres \\
\hline Empleado & 41 & 34 & & 30 & 30 \\
Desempleado & 59 & 51 & & 64 & 56 \\
Inactivo & 44 & 53 & & 47 & 61 \\
\hline
\end{tabular}

FUENTE: Elaboración propia con base en la Encuesta Nacional de Ingreso y Gasto de los Hogares 2008.

haya brecha de género en esta categoría. Ello demuestra que el trabajo resulta una variable fundamental para evitar que las personas se encuentren en pobreza. En las tasas de pobreza de las mujeres empleadas y de las desempleadas o inactivas se advierte una diferencia sustancial. Otra cuestión que llama la atención es que bajo ambos modelos las tasas de pobreza de las mujeres desempleadas resultan inferiores a las de los hombres, presumiblemente porque cuando la mujer está desempleada tiene la posibilidad de contar con el ingreso de la pareja; al revés no necesariamente ocurre así, dado que el hombre suele ser el principal sostenedor del hogar y con frecuencia esto implica que su pareja femenina no trabaje. A pesar de que, como se mencionó, cuando las personas son desempleadas o inactivas aumenta para ambos géneros su tasa de pobreza al pasar del modelo unitario al modelo colectivo, la brecha de género se hace mucho mayor en el caso de las mujeres inactivas bajo el modelo colectivo.

En resumen, podemos apreciar que el rostro de la pobreza femenina en México se transforma cuando se pasa del modelo unitario al modelo colectivo porque sus tasas de pobreza se suelen elevar en relación con la gran mayoría de las variables descritas, y la brecha de género entre hombres y mujeres se vuelve pronunciada. Esto es totalmente claro en términos de localización geográfica, etnicidad y edad.

En el caso de la educación, curiosamente también se incrementa la brecha de género, a pesar de que para los niveles más altos la tasa de pobreza femenina no aumenta al pasar al modelo colectivo. El único caso en que no se cumple la regularidad de que la brecha de género aumenta es en relación con el estatus ocupacional, pues bajo 
el modelo colectivo la tasa de pobreza de hombres y mujeres empleados es idéntica, e incluso la tasa de pobreza de las mujeres baja al pasar del modelo unitario al modelo colectivo.

Ante lo anterior podemos concluir que los resultados de las estadísticas descriptivas muestran que existe una brecha de género importante en la pobreza que no puede ser despreciada y que queda totalmente escondida en el modelo unitario de hogar. Ello implica que bajo el modelo colectivo, a igual condición en otra división social, ser mujer suma una mayor desventaja social a la situación de pobreza, de modo que las mujeres viven más en la pobreza que su contraparte masculina. También los resultados muestran que el estatus ocupacional desempeña un papel fundamental para aminorar o incrementar tales desventajas, pues el estatus ocupacional es la única categoría entre las analizadas donde la situación de la mujer se comporta de manera muy similar a la de los hombres. Estos hallazgos manifiestan que si hubiera una paridad de género en la participación laboral, la situación de pobreza de las mujeres disminuiría sustancialmente.

\section{La evidencia sobre la pobreza femenina en México usando el modelo colectivo}

Hasta aquí se ha descrito cómo cambia la pobreza femenina de acuerdo con distintas variables al pasar del modelo unitario al modelo colectivo, usando estadísticas descriptivas. En general se encuentra que las tasas de pobreza femeninas son mayores bajo el modelo colectivo, al igual que la brecha de género entre hombres y mujeres (con algunas excepciones). Muchas de las características anteriormente mencionadas se interrelacionan unas con otras. Por ejemplo, las posibilidades de ocupación de las mujeres están altamente relacionadas con su nivel educacional, si bien la posibilidad de obtener cierto nivel educacional podría estar limitada por la condición de etnicidad. En este apartado se construye un modelo de regresión logística para encontrar cuáles son los factores que más peso tienen en la explicación de la pobreza femenina bajo el modelo colectivo. La ventaja de usar el análisis multivariado es que permite explorar el peso específico de cada variable mientras se controla el impacto de las otras.

Para clarificar los resultados de la regresión se calcularán posteriormente las probabilidades de estar en pobreza de dos mujeres hi- 
potéticas. En el primer caso se trata de una mujer adulta en edad laboral, y en el segundo caso se trata de una mujer de la tercera edad. Este ejercicio resulta muy útil porque permite variar solamente una característica a la vez, manteniendo todas las demás constantes. De este modo puede compararse fácilmente el peso que los distintos factores tomados en cuenta en el modelo tienen sobre la pobreza femenina en México.

El modelo fue aplicado únicamente a las mujeres mayores de 18 años (sin incluir hombres o menores de edad) porque lo que se pretende explicar es la pobreza femenina. Sin embargo las características de las mujeres relacionadas con sus parejas y con sus hijos fueron incluidas como variables en el modelo. En consecuencia, la variable de interés (dependiente) es ser mujer adulta y estar en pobreza bajo el modelo colectivo de hogar con la asunción de una retención del 20 por ciento.

Las variables independientes se integraron para intentar englobar la influencia de las tres instituciones que determinan el acceso a los recursos en la sociedad y que fueron abordadas en el segundo apartado. Para poder medir la importancia de la participación en el mercado laboral se incluyeron cinco variables binarias que dan cuenta de la condición de actividad de la mujer. Se seleccionó la categoría de inactiva como base y en ésta se englobó a todas aquellas que no participan en el mercado laboral, pero sin incluir a las pensionadas. La segunda categoría que se consideró correspondió a todas aquellas mujeres que declararon en la encuesta que eran jubiladas o pensionadas. Se consideró importante separar esta categoría porque permite comparar la situación relativa de las mujeres de la tercera edad pensionadas con el resto de las inactivas. La tercera categoría incluida abarca a todas las mujeres que se declaran desempleadas y manifiestan que buscan trabajo. La cuarta categoría comprende a todas aquellas empleadas en negocios informales (es decir, que no cuentan con contrato laboral o prestaciones) y a todas las que se declaran trabajadoras por cuenta propia o dueñas de negocios pero que no cuentan con registro fiscal. Por último se incluye en la categoría de trabajadoras formales a todas aquellas que son empleadas y cuentan con contrato laboral y prestaciones, y a las que trabajan por cuenta propia o son dueñas de un negocio con registro fiscal. También en este último caso se consideró pertinente separar la situación laboral formal de la informal para obtener más información sobre las diferencias entre las mujeres empleadas de acuerdo con la calidad de su empleo. 
Para determinar la influencia de la política social se incluye, en primer lugar, una variable binaria que denota el acceso a la seguridad social, lo cual implica tanto el acceso a recursos, como a los servicios básicos tales como la salud y en algunos casos a los servicios de cuidado como las guarderías. Asimismo se incluyen ocho variables binarias que dan cuenta del acceso a otro servicio básico importantísimo relacionado con la política social: la educación. Las variables denotan el último nivel de estudios terminado y van desde la primera variable (base) que caracteriza una situación de analfabetismo, hasta la octava, que corresponde a la persona que terminó un posgrado.

Asimismo, para determinar la posible influencia de la familia en el bienestar femenino se incluyó una serie de variables que denotan los patrones de convivencia y las características demográficas del hogar. Se consideró en primer lugar una variable que denota la edad de la persona. Posteriormente se incluyó una variable binaria que indica si la persona tiene pareja (ya sea casada o en unión libre). También se incorporó una variable numérica que indica el número de menores en el hogar. Por último se consideró importante conocer si en el hogar radican otros adultos más allá de la familia nuclear, y cuál es su influencia en la pobreza femenina; para ello se construyó una variable numérica con el número de otros adultos.

Asimismo se incluyen en el modelo otras dos variables que ya fueron usadas en las estadísticas descriptivas y que representan divisiones sociales importantes. Por un lado, se incluyó una variable binaria que denota la pertenencia a un grupo indígena, entendiendo ésta como todas aquellas mujeres que declaran hablar lengua indígena. En segundo lugar, se incluyó una variable binaria que denota si la persona vive en un área urbana. Las áreas urbanas fueron definidas como aquellas que cuentan con más de 2500 habitantes, para estar en concordancia con la Línea de Bienestar de Coneval que las define de igual manera. En el cuadro 9 se presenta el modelo.

El modelo revela que la edad ${ }^{17}$ tiene una relación inversa con la pobreza femenina, pues a mayor edad, menor pobreza. Sin embargo, su relación es bastante débil, lo cual resulta lógico ya que la situación de personas con edades similares puede resultar muy diferente porque depende de muchas otras características.

17 Se introduce la variable edad al cuadrado para comprobar si el efecto de la edad en la pobreza se sostiene también para los ancianos. Al resultar insignificante este término no lineal puede afirmarse que el efecto de la edad en la pobreza no cambia en el extremo. 


\section{CUADRO 9}

Regresión logística que predice la pobreza femenina bajo el modelo colectivo usando la Línea de Bienestar de Coneval (2008)

\begin{tabular}{lc}
\hline Variables independientes & Estar en pobreza \\
\hline Edad & $-0.044^{* *}$ \\
Edad $^{2}$ & $0.000^{* *}$ \\
Con pareja & 0.018 \\
Número de menores en el hogar & $0.206^{* *}$ \\
Número de otros adultos en el hogar & $-0.092^{* *}$ \\
Perteneciente a grupo indígena & $0.466^{* *}$ \\
Localización urbana & $0.235^{* *}$ \\
Estatus económico inactiva (base) & \\
Estatus económico pensionada & $-1.093^{* *}$ \\
Estatus económico desempleada & 0.044 \\
Estatus económico trabajadora informal & $-0.861^{* *}$ \\
Estatus económico trabajadora formal & $-1.820^{* *}$ \\
Nivel de educación sin instrucción (base) & \\
Nivel de educación alfabetismo & $-0.374^{* *}$ \\
Nivel de educación primaria completa & $-0.748^{* *}$ \\
Nivel de educación secundaria completa & $-1.115^{* *}$ \\
Nivel de educación preparatoria completa & $-1.605^{* *}$ \\
Nivel de educación técnica & $-1.584^{* *}$ \\
Nivel de educación licenciatura & $-2.396^{* *}$ \\
Nivel de educación posgrado & $-3.513^{* *}$ \\
Acceso a la seguridad social & $-0.831^{* *}$ \\
Constante & $2.450^{* *}$ \\
$\mathrm{R}^{2}$ & 0.21 \\
\hline & \\
\hline & \\
\hline
\end{tabular}

$* * \mathrm{p}<0.01$

NOTA: El modelo fue calculado usando el factor de expansión de la ENIGH. El número de observaciones sin el factor de expansión es $N=76301$.

FUENTE: Elaboración propia con base en la Encuesta Nacional de Ingreso y Gasto de los Hogares 2008. 
En términos de convivencia tener pareja tiene una relación positiva con la pobreza, aunque tal relación es baja. Ello implica que las mujeres que viven en hogares encabezados por hombres tienden a ser más pobres que las que encabezan sus propios hogares. Este resultado es congruente con lo que muestran las estadísticas descriptivas (cuadro 2), aunque puede resultar sorprendente, por lo que se explica a continuación.

En primer lugar se encuentra que cuando las mujeres tienen pareja, su tasa de participación laboral baja sustancialmente como consecuencia de sus obligaciones familiares, con respecto a las mujeres que no tienen pareja; tal y como se muestra en el cuadro 10.

Otros autores ya se han referido previamente a esta situación. Rodríguez (2010) afirma que sin importar la edad, en México una pareja tiende a resultar más pobre que una persona soltera. Ello debido a que es menor la participación en el mercado laboral de las mujeres casadas o en pareja, lo que implica que en muchos casos la pareja viva de un solo ingreso. En el caso de los hogares encabezados por mujeres se encuentra que generalmente cuentan con un ingreso propio, ya sea porque ellas trabajen o porque reciban transferencias de otros hogares en forma de pensiones alimentarias o de remesas (Villareal y Shin, 2008). A su vez, este último tipo de hogar tiene un miembro menos (la pareja masculina), por lo que el ingreso debe de mantener a menos personas. El grueso de las madres solteras como consecuencia de embarazos indeseados no se encuentra ahí, ya que al no contar con un ingreso independiente se quedan en el hogar familiar (Rodríguez, 2010: 234-235). Es por eso que se encuentra que los hogares ampliados sufren mayor pobreza que los nucleares (Damián, 2008; Rodríguez, 2010). De hecho, tal como lo comprueban Villareal y Shin (2008: 569) cuando analizan la situación de los hogares encabezados por mujeres con menores, ${ }^{18}$ el $80 \%$ de estos hogares está encabezado por mujeres que son viudas o divorciadas y sólo el $8 \%$ corresponde a madres solteras. Las mujeres que encabezan sus propios hogares tienen fuentes de ingreso independientes, ya sea porque cuenten con una pensión de la seguridad social o porque reciben transferencias de dinero provenientes del padre. Ambos casos dan muestra de que cuando las políticas protegen el derecho de las madres

${ }^{18}$ Los autores se refieren solamente a este tipo de hogares excluyendo a aquellos hogares encabezados por mujeres solas o aquellos hogares donde hay una pareja presente. Ello para que los hogares encabezados por mujeres puedan ser comparables con los hogares nucleares encabezados por hombres. 
Rodríguez, UNA CARACTERIZACIÓN DE LA POBREZA FEMENINA

CUADRO 10

Tasa de participación en el mercado laboral de las mujeres mayores de 18 años con y sin pareja (2008)

\begin{tabular}{lcc}
\hline & Sin pareja & Con pareja \\
\hline Activas & 56 & 38 \\
Inactivas & 44 & 62 \\
\hline
\end{tabular}

FUENTE: Elaboración propia con base en la Encuesta Nacional de Ingreso y Gasto de los Hogares 2008.

a contar con un ingreso para el cuidado de sus hijos, éstas se encuentran en una mejor situación que aquellas madres que no cuentan con tal protección.

No sorprende que el modelo del cuadro 9 describa que el número de menores en el hogar también tiene una relación positiva con la pobreza, lo cual resulta lógico porque los menores no cuentan con recursos propios, por lo que su presencia incrementa las necesidades y los gastos del hogar; a su vez, las mujeres tienen mayores probabilidades de dejar de trabajar para dedicarse a su cuidado. El número de otros adultos en el hogar tiene una relación negativa con la pobreza, lo que significa que su presencia contribuye a reducirla, siendo su coeficiente parecido al efecto que provoca la presencia de la pareja, pero en sentido inverso. Es decir, la pareja aumenta la pobreza de las mujeres y la presencia de otros adultos la baja. Ello puede deberse a que la presencia de otros adultos en el hogar tiende a incrementar sus ingresos, o ayuda en las labores de cuidado de los hijos, y ofrece por tanto mayores probabilidades de que la mujer deje de participar en el mercado laboral.

Por otro lado, pertenecer a un grupo indígena tiene una clara y fuerte relación con la pobreza; sin embargo vivir en una localidad urbana tiene una relación positiva con la pobreza femenina. Este resultado es sorpresivo, ya que las tasas de pobreza de las mujeres en el ámbito rural resultan sustancialmente más elevadas que en el medio urbano, como se muestra en el cuadro 4. Sin embargo el resultado podría deberse al hecho de que la mayoría de las mujeres en pobreza viven en localidades urbanas. El cuadro 11 muestra el porcentaje de todas las mujeres y de las mujeres en pobreza que viven en localidades urbanas y rurales. Como puede apreciarse, el $73 \%$ de las mujeres en pobreza viven en localidades urbanas. Otra explicación posible podría tener que ver con el hecho de que la línea de pobreza usada por 
Coneval para el ámbito rural es menor que la línea de pobreza urbana. El modelo podría estar reflejando estos efectos. ${ }^{19}$

El estatus económico también muestra una relación con la pobreza que no resulta sorprendente. Todas las categorías con excepción de la de desempleada tienen una relación negativa con la pobreza en relación con la categoría base, que es inactiva; la de desempleada sí tiene una relación positiva. Por tanto, como podría esperarse, el estatus económico que más pobreza genera es el de inactivo seguido por el de desempleado. La categoría que más contribuye a no estar en pobreza es la de trabajadora en el sector formal; la segunda categoría que tiene una relación inversa más fuerte con la pobreza es la de pensionada, y luego siguen las trabajadoras informales. Curiosamente las pensiones tienden a bajar la pobreza mucho más que ser trabajadora en el sector informal.

Respecto a la educación, no existe ninguna sorpresa. A medida que aumenta el nivel educacional va aumentando sustancialmente su relación inversa con la pobreza. Ello en oposición a la categoría base, que es sin instrucción. Por último, el modelo muestra claramente que acceder a la seguridad social también tiene una importante relación inversa con la pobreza.

El cuadro 12 muestra la predicción de probabilidades de estar en pobreza para una mujer adulta hipotética bajo el modelo colectivo, usando la regresión del cuadro 9. Para realizar este tipo de ejercicio resulta conveniente escoger como base del modelo aquellas características que sean más o menos representativas de la población pero que no presenten una probabilidad muy alta de estar en pobreza. Posteriormente comienza a variarse solamente una característica a la vez, mientras el resto se mantiene constante. La ventaja de este ejercicio es que se pone a cada una de las variables en igualdad de condiciones y permite dilucidar la importancia que puede tener cada una por separado para disminuir o agravar la pobreza.

La primera información que podemos extraer del cuadro 12 es que la mujer hipotética tiene 35 años, no es indígena, vive sola en un área urbana, tiene un empleo en el sector formal de la economía y

19 Se elaboró un modelo adicional, idéntico al del cuadro 9, que no se presenta aquí. Se modificó solamente la variable dependiente, que en este caso fue estar en pobreza bajo el modelo unitario. Dicho modelo mostró resultados similares ya que allí también vivir en un ámbito urbano tenía una relación positiva y significativa con la pobreza, lo cual implica que este efecto no se debe al hecho de usar el modelo colectivo, sino que presumiblemente tiene que ver más bien con los elementos ya mencionados. 
Rodríguez, UNA CARACTERIZACIÓN DE LA POBREZA FEMENINA

CUADRO 11

Porcentaje de todas las mujeres y de las mujeres en pobreza bajo el modelo colectivo en localidades rurales y urbanas (2008)

\begin{tabular}{lcc}
\hline & $\begin{array}{c}\text { Porcentaje } \\
\text { de todas las mujeres }\end{array}$ & $\begin{array}{c}\text { Porcentaje de mujeres en pobreza } \\
\text { bajo el modelo colectivo }\end{array}$ \\
\hline Rural & 20 & 27 \\
Urbano & 80 & 73 \\
Total & 100 & 100 \\
\hline
\end{tabular}

FUENTE: Elaboración propia con base en la Encuesta Nacional de Ingreso y Gasto de los Hogares 2008.

acceso a la seguridad social, y que su nivel educacional es de secundaria (que es el último nivel que el Estado garantiza de manera universal). Con estas características la probabilidad de estar en pobreza de dicha mujer es de 9.1. Cuando en vez de ser soltera la mujer es casada, su probabilidad de pobreza sube ligeramente a 9.3. Si en el hogar de la mujer hipotética hubiera menores, la presencia de éstos sube el riesgo de pobreza notablemente pasando de 11 si es uno, a 19 si son cuatro. Por el contrario, la presencia de otro adulto en el hogar disminuye la probabilidad de pobreza a 8.4. La condición de etnicidad sube sustancialmente sus probabilidades de pobreza a 14. Si la mujer de referencia en vez de vivir en un área urbana habita en una rural sus probabilidades de pobreza bajan. Este resultado debe de ser tomado con cuidado porque puede estar reflejando los efectos que ya se describieron, ya que las tasas de pobreza en el ámbito rural son mucho más elevadas que en el ámbito urbano. El estatus económico influye marcadamente en la pobreza. Si la mujer en vez de estar empleada en el sector formal lo estuviera en el informal su probabilidad de pobreza subiría a 20. En cambio, si fuera inactiva o desempleada sus probabilidades de pobreza se elevarían a 39. El nivel educacional también resulta fundamental para las probabilidades de pobreza ya que va desde una probabilidad de 23 si esta mujer hipotética no tuviera ninguna educación, hasta una probabilidad casi despreciable de 0.9 si la misma mujer contara con posgrado. Por último, si la mujer no tuviera acceso a la seguridad social, la probabilidad se incrementaría a 19.

El cuadro 13 muestra la predicción de probabilidades de estar en pobreza para una mujer de la tercera edad bajo el modelo colectivo, usando la regresión del cuadro 9. 


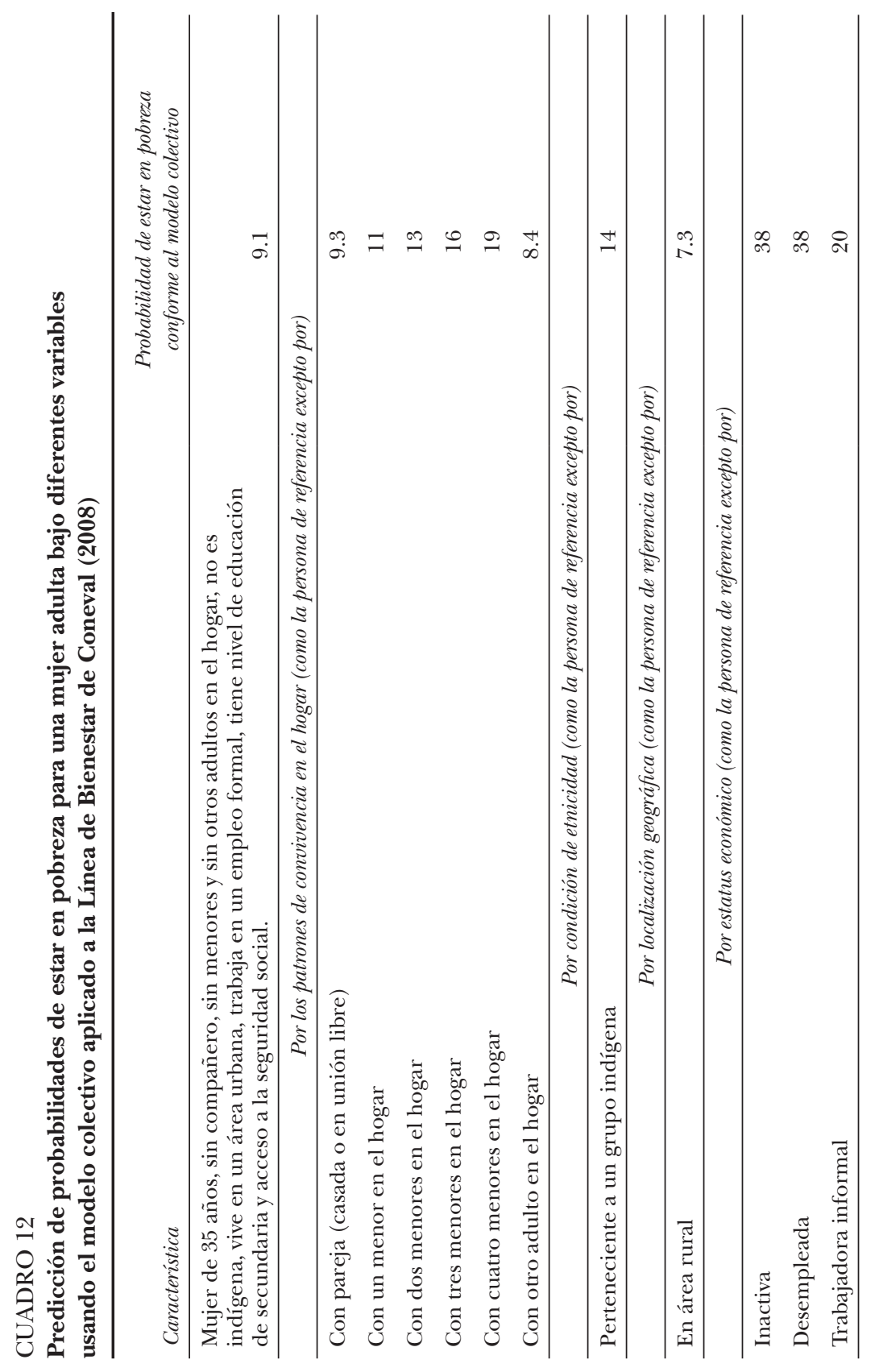




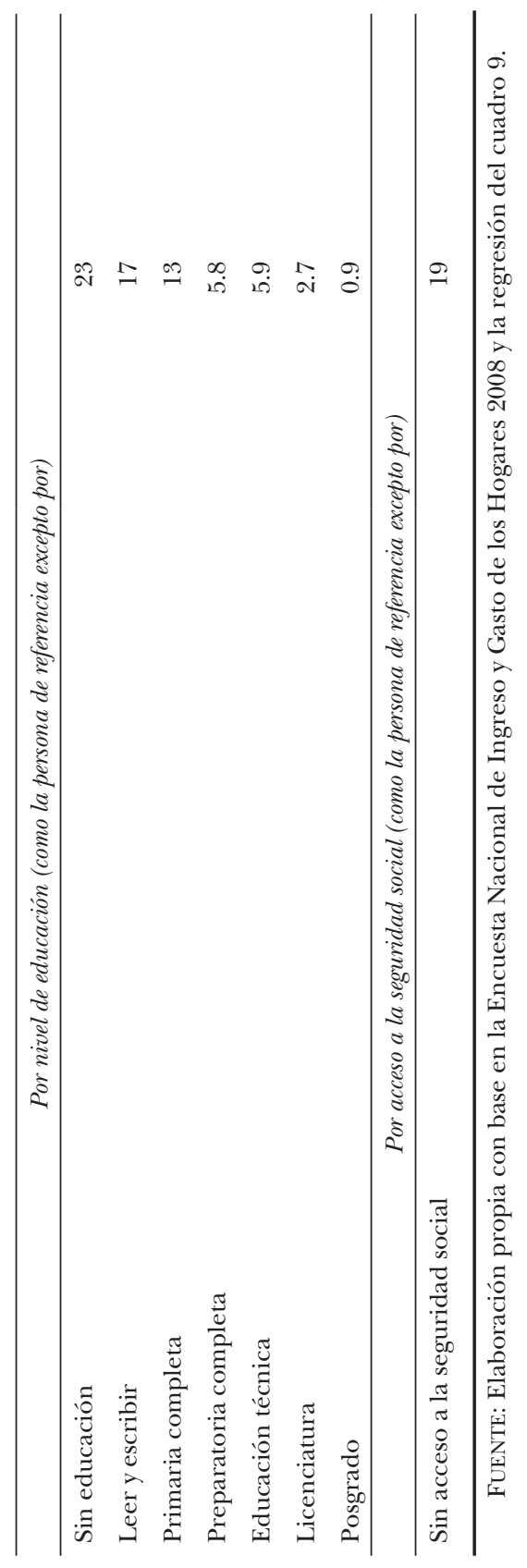




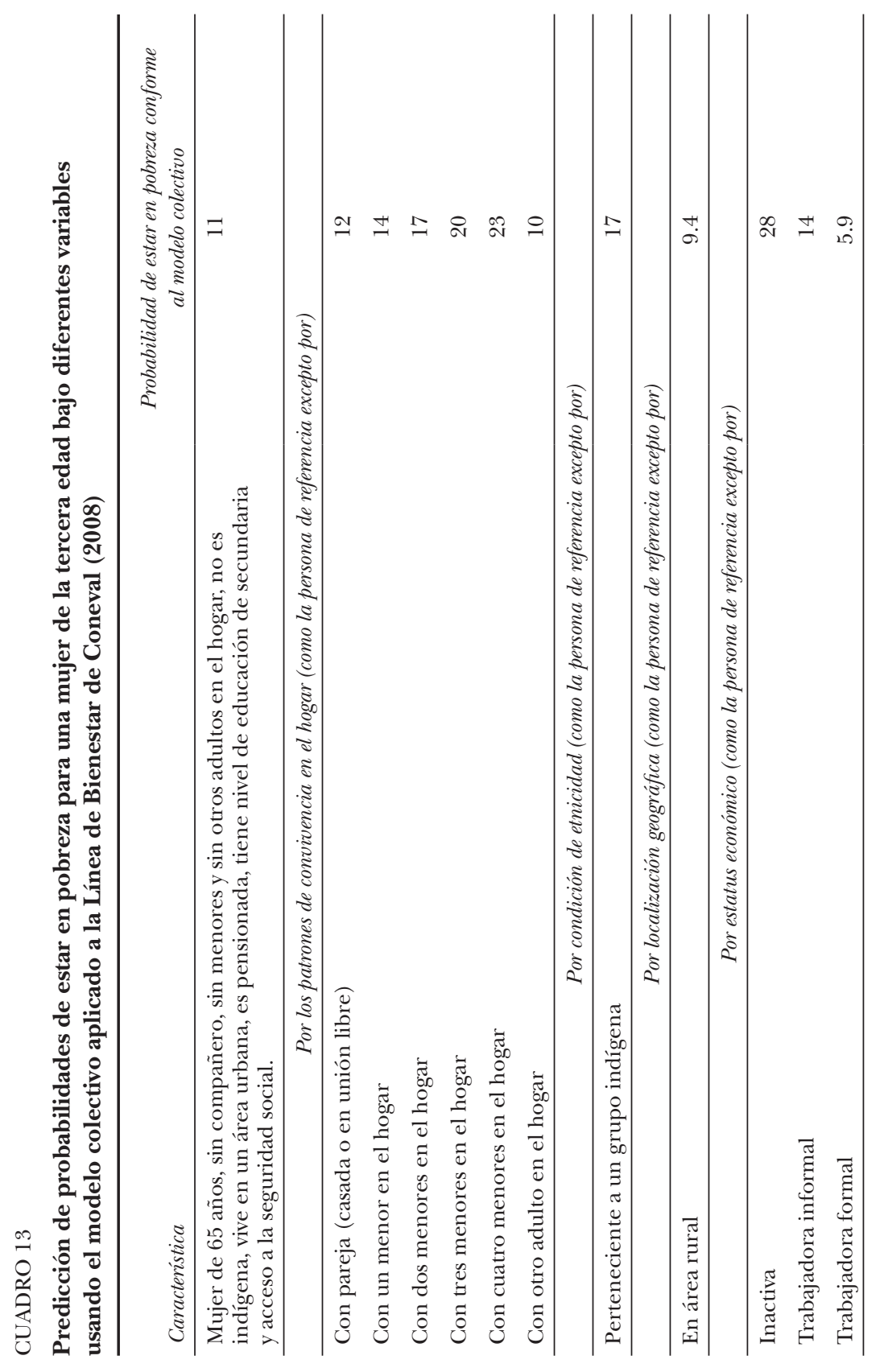




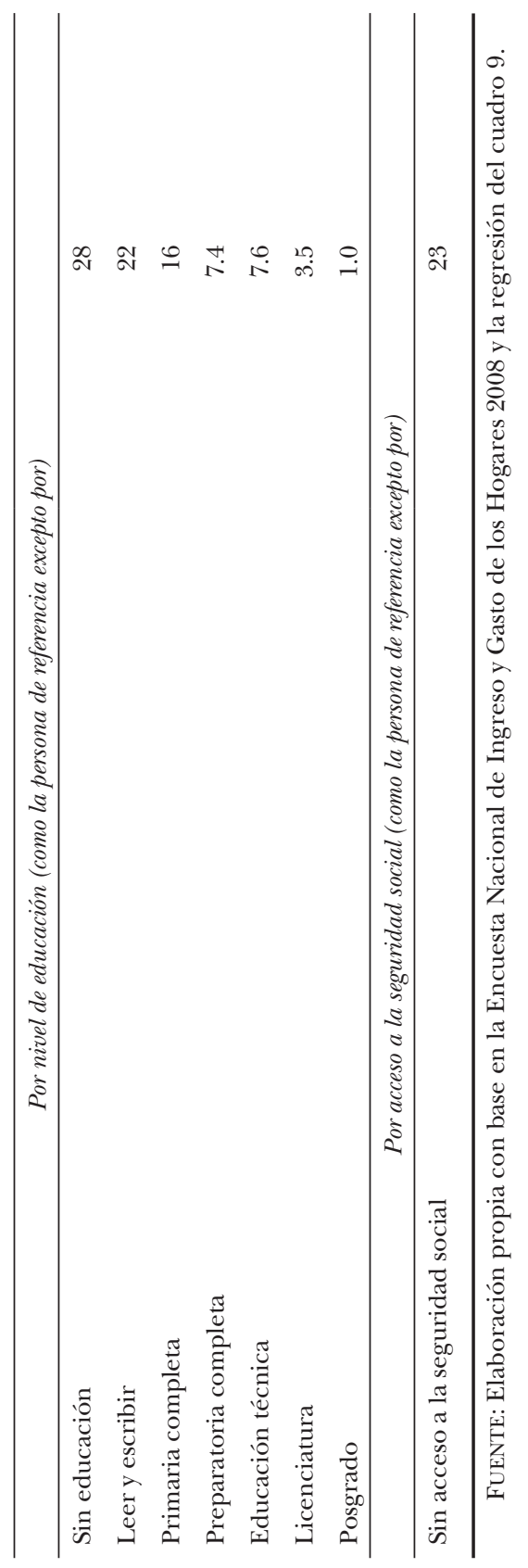


Las características de la mujer hipotética, al igual que en el cuadro 12 , intentan ser representativas pero a la vez no presentan una probabilidad muy alta de estar en pobreza. En este caso se seleccionó a una mujer de 65 años que vive sola, no es indígena, habita en un área urbana, es pensionada, tiene nivel de educación secundaria y acceso a la seguridad social. Con estas características tiene una probabilidad de 11 de estar en pobreza. La primera cuestión interesante es que una mujer pensionada de 65 años tiene más probabilidades de estar en pobreza que una mujer de 35 años que trabaja. Cuando en vez de ser soltera la mujer vive con su pareja, sus probabilidades de pobreza se incrementan ligeramente a 12. Si conviviera con menores en el hogar incrementa en 3 puntos sus probabilidades de pobreza por cada menor. En cambio la presencia de otros adultos en el hogar contribuye a disminuir sus niveles de pobreza. Si la mujer hipotética en cuestión fuera indígena, sus probabilidades de pobreza aumentan en 6 puntos. Si viviera en un área rural disminuyen por las mismas razones que se explicaron en el caso anterior. El estatus ocupacional resulta sumamente importante, ya que si ella fuera inactiva en vez de ser pensionada las probabilidades de pobreza aumentan hasta 28; si no fuera pensionada y trabajara en el sector informal sus probabilidades también aumentarían a 14 , lo que implica que para las mujeres de la tercera edad una pensión es más importante para no estar en la pobreza que sobrevivir del sector informal. En cambio si la mujer no estuviera pensionada y todavía trabajara en el sector formal, sus probabilidades de pobreza disminuirían sustancialmente. El nivel educacional también es muy relevante, dado que si la mujer no tuviera ninguna educación sus probabilidades de estar en pobreza aumentarían hasta 28, pero en cambio si tuviera nivel de posgrado, éstas bajarían hasta 1. Por último, encontramos que la falta de acceso a la seguridad social eleva sus probabilidades de pobreza hasta 23 .

\section{El peso de las distintas causas de la pobreza femenina conforme al modelo colectivo de hogar}

Como se expuso anteriormente, en la sociedad contemporánea las tres instituciones que median el acceso al ingreso de las personas son el mercado laboral, la política social y la familia. En la interacción de las tres se determinan las probabilidades de estar en pobreza de los distintos grupos sociales. En esta parte se usan los resultados obtenidos 
en esta investigación como la base para analizar la interrelación de estas tres instituciones y su contribución a la pobreza femenina. Cuando es necesario se usan también los hallazgos de otros estudios relevantes sobre el tema, para aclarar o profundizar en ciertos aspectos de los resultados obtenidos.

Respecto al mercado de trabajo se encuentra que para las mujeres adultas el estatus económico resulta el factor más importante para determinar sus probabilidades de estar en pobreza. Ello porque su condición de ocupación es la variable más influyente de todas las consideradas en el modelo, ya que dependiendo de cuál sea ésta las probabilidades de pobreza pueden incrementarse en 29\% (de 9.1 si es empleada formal a 38 si no trabaja) (cuadro 12). De hecho, entre todos los factores tomados en consideración en el modelo, la condición de inactividad o desempleo genera las mayores probabilidades de estar en pobreza. Por el contrario, cuando la mujer está empleada su tasa de pobreza es igual a la de los hombres (cuadro 8), y en este caso desaparece totalmente la brecha de género que existe para todas las demás variables que han sido tomadas en cuenta en esta investigación.

No obstante, las posibilidades de que el empleo sea efectivo para evitar la pobreza femenina dependen de la calidad del mismo. Como puede apreciarse en el cuadro 12, si la mujer hipotética en vez de participar en el mercado formal lo hiciera en el informal, sus probabilidades de pobreza se elevan sustancialmente, aunque se mantienen claramente por debajo de las probabilidades de pobreza si la mujer estuviera fuera de la fuerza laboral.

De hecho, de acuerdo con Costa y Silva (2010), de los tres factores que contribuyen a la desigualdad de género en el mercado laboral (menor participación laboral de la mujer respecto a los hombres; mayores tasas de informalidad y desempleo para las mujeres respecto a los hombres y salario inferior al masculino en igualdad de condiciones), el que mayor influencia puede tener en la caída de la pobreza en el caso de México es el primero; es decir, que las tasas de participación femenina se igualaran a las masculinas.

No obstante, la participación laboral de las mujeres adultas es sustancialmente menor que la de los hombres. Mientras que en el año 2008 el $81 \%$ de los hombres mayores de 18 años participaba en el mercado laboral, sólo el 44\% de las mujeres lo hacía (Rodríguez, 2012), lo cual representa prácticamente la mitad de la de los hombres. Las tasas de participación femenina en México son bajas incluso si se comparan con las de otros países de América Latina (CEPAL, 2008: 39). 
Sin embargo, el tema de la incorporación al mercado laboral también tiene que ser tomado con cuidado, ya que no necesariamente es una panacea para resolver el problema de pobreza de las mujeres. El trabajo formal, tal como hoy existe, las discrimina al no reconocer la carga reproductiva, la de trabajo doméstico y la de cuidado (CEPAL, 2009: 49), que recae desproporcionadamente en ellas. El mercado de trabajo tiene una estructura que favorece a los hombres por encima de las mujeres porque existe una serie de barreras al empleo femenino, como por ejemplo horas inflexibles, inadecuada capacidad en las guarderías para el cuidado de los hijos, y corta jornada en la educación básica de los niños. Todos estos factores hacen que la incorporación al mercado laboral sea muy desventajosa para ellas. No es suficiente mejorar la tasa de ocupación de la mujer, sino que es necesario reforzar su posición dentro del mercado laboral, particularmente en su segmento más bajo (Lister, 2010: 170).

Respecto a los efectos de la política social, en el modelo se encuentra, en primer lugar, que el acceso a la seguridad social resulta sumamente importante para evitar la pobreza, ya que las probabilidades de pobreza aumentan sustancialmente ( $10 \%$ en el caso de la mujer adulta) si no se cuenta con dicho acceso. En el caso de México ello significa que la mujer no cuenta con cobertura universal de salud ${ }^{20} \mathrm{ni}$ con el derecho a un ingreso que compense ciertos riesgos, como la invalidez, la maternidad o el envejecimiento, pues el acceso a la seguridad social está totalmente vinculado a la participación en el mercado formal. No existen apoyos universales sobre la base de ingresos fiscales que permitan a las mujeres que no participan en la seguridad social contributiva contar con el derecho a la obtención de ingresos por su condición de madres. Tampoco se cuenta con apoyos universales para las mujeres que les permitieran incrementar su participación en el mercado laboral brindando servicios de cuidado en guarderías o de atención a otros miembros del hogar como ancianos o discapacitados. De hecho, en general en América Latina la tasa de participación laboral de las mujeres que tienen hijos de hasta 6 años a su cargo está en los quintiles más pobres, notablemente más baja que la de las mujeres del mismo nivel socioeconómico sin menores o con niños mayores de esa edad. Sin embargo esta diferencia desaparece en el quintil más alto, lo cual

20 Aunque el objetivo del Seguro Popular es incluir a todas las personas cuyo acceso a la salud no está cubierto por la seguridad social formal, no constituye un derecho similar al que brinda la seguridad social, pues no cubre todas las enfermedades, sino solamente un paquete básico. 
implica que las más pobres pagan un costo adicional al tener hijos, porque la ausencia de servicios públicos les dificulta su participación laboral (CEPAL, 2009: 45).

El acceso a la seguridad social cobra particular relevancia en el caso de las mujeres de la tercera edad. De hecho, en este caso, la importancia crucial del mercado laboral para evitar la pobreza se sustituye por el acceso a la seguridad social. Las mujeres de la tercera edad sin acceso a la seguridad social aumentan su riesgo de pobreza en $12 \%$. Lo único que podría colocarlas en una situación mejor sería continuar trabajando en el sector formal. Sin embargo en la tercera edad las capacidades para participar en el mercado laboral disminuyen, así como las posibilidades de encontrar un empleo, por cuestiones de discriminación. Ante esta situación, si la mujer no cuenta con una pensión se encuentra totalmente desamparada. Como puede constatarse en el cuadro 13, incluso si trabajara en la informalidad, sus probabilidades de pobreza serían superiores a las de la mujer pensionada.

La expansión de los programas sociales que se ha dado en los últimos años en México no ha sido por la vía de ampliar la cobertura universal a la seguridad social, sobre la base de fortalecer los derechos sociales; sino mediante los programas de transferencias monetarias condicionadas. En particular el programa Oportunidades opera sobre la base de transferir pequeñas cantidades de dinero (muy inferiores a la medida de pobreza oficial de Coneval) a un sector de los pobres, condicionado a que lleven a sus hijos a la escuela y asistan a los centros de salud. Este programa, que cubre a 5 millones de familias, entrega las transferencias monetarias directamente a las mujeres. Es presumible que contribuya a incrementar los recursos que utilizan las mujeres pobres para manejar sus hogares, sin embargo esto no necesariamente repercute en un beneficio directo para la propia mujer. Al ser la mujer el activo más importante de la política, se le pide que desempeñe funciones que tradicionalmente se asocian a su condición de género, tales como el manejo económico en situaciones de carencia, la realización cotidiana de trabajo no remunerado y su responsabilidad como cuidadora de otros miembros del hogar. Estas iniciativas refuerzan el rol tradicional de la mujer y recargan aún más el intensivo trabajo asociado con su situación de pobreza (Chant, 2010). En este sentido, cuando la prioridad de los gobiernos y los organismos internacionales es aliviar la pobreza en general, el papel otorgado a las mujeres en estos programas responde más al pragmatismo que a un intento de lograr equidad de género (Molyneux, 2006a). Ello porque 
los programas tal como están concebidos no garantizan un mejor acceso al mercado laboral para ellas, ni tampoco una seguridad económica, dado que tienen un carácter finito porque dejan de transferir fondos cuando se cumplen ciertas condiciones (por ejemplo, cuando los hijos acaban la secundaria), no importando si el hogar sigue o no en pobreza. No se trata de un derecho de la mujer a obtener recursos.

Otra de las variables relacionadas con la política social cuya influencia en la pobreza es muy importante es el nivel educacional. De hecho, en orden de importancia, ésta es la segunda variable más influyente para determinar la pobreza femenina, después del estatus ocupacional. La educación varía las probabilidades de pobreza en un rango de $22 \%$ para la mujer adulta. En los niveles educacionales más bajos la pobreza resulta muy elevada, y en los más altos su probabilidad es prácticamente inexistente. En términos de política esto implica que la educación es un antídoto importante contra la pobreza femenina, por lo que garantizar el acceso de las mujeres a los niveles educativos más altos puede resultar fundamental. Su influencia en la disminución de los niveles de pobreza podría radicar presumiblemente en dos razones, a pesar de que como se expuso anteriormente, a igual nivel de escolaridad los salarios de las mujeres son inferiores que los de los hombres. Primero, porque cuanto mayor sea su nivel de educación, más posibilidades tendrán de contar con un ingreso independiente derivado de un mejor empleo. En segundo lugar, porque con una mayor educación las mujeres tendrán más conocimiento de sus posibilidades de escoger alternativas de vida que las liberen de los patrones tradicionales que las conducen a la pobreza, como por ejemplo, que sean capaces de planear concienzudamente su reproducción (Ordorica, 2010: 36). La influencia de la variable educación también resulta relevante para las mujeres de la tercera edad. Como se evidencia, no sólo resulta importante para determinar los ingresos por trabajo, en el caso de la mujer adulta, sino también para determinar los niveles de pensión a los que se puede acceder posteriormente. Ello porque en el modelo de la mujer hipotética de la persona de la tercera edad, se trata de alguien que ya no participa en el mercado laboral, sin embargo el nivel educacional es la variable que más hace oscilar su probabilidad de estar en pobreza (27 por ciento).

Un último efecto, que tiene una gran relación con el ámbito de la política social, es la condición de etnicidad. Por sí misma eleva la probabilidad de pobreza (alrededor de 6\%) asumiendo que el resto de las condiciones se mantienen constantes. Ello habla de las grandes 
desventajas que todavía persisten en la sociedad para los grupos indígenas. Es por ello que desde la política social se necesitan tomar medidas compensatorias que logren una mayor equidad para este grupo social y equiparen sus oportunidades con las de las no indígenas.

Asimismo podemos extraer del modelo los efectos de la familia en la pobreza femenina. Se aprecia que los hijos incrementan sustancialmente el riesgo de la pobreza femenina y que es tanto más alto cuanto mayor es el número de hijos (con 4 hijos aumenta alrededor de 10\%). El efecto de los hijos en la pobreza femenina se torna explicable porque ellos incrementan las necesidades del hogar sin que aumenten los ingresos. Por otro lado, cuando se trata de hogares extensos, la presencia de otros adultos tiene un efecto positivo porque disminuye el riesgo de pobreza de las mujeres debido a que contribuyen al hogar con una fuente de ingreso adicional o con ayuda en las labores de cuidado, de modo que la mujer tiene más oportunidades de participar en el mercado laboral. Este efecto contrasta con el que provoca la presencia de la pareja masculina.

Cuando las mujeres viven en pareja su riesgo de pobreza aumenta ligeramente, ya que como se demostró anteriormente, tienen menor participación en el mercado laboral que las mujeres sin pareja. Sólo $38 \%$ de las mismas participa (cuadro 10). Ello se debe, en primer lugar, a que las mujeres con pareja tienen más tendencia a reproducirse que las mujeres que no tienen pareja. Como se mencionó, este proceso resulta particularmente complicado para las mujeres más pobres porque no cuentan con la provisión de servicios públicos necesarios que las apoyen en las labores de cuidado de los hijos, de modo que puedan participar en el mercado de trabajo. Además, cuando las mujeres tienen pareja aumenta su carga de trabajo reproductivo en el hogar. De acuerdo con la CEPAL, los hombres en México, sin importar su quintil de ingreso, destinan muy poco tiempo a las labores reproductivas del hogar, con lo cual se demuestra que los hombres son bastante inflexibles respecto a su participación en las labores del hogar, independientemente de su nivel económico. En el caso de las mujeres se observa que a medida que aumenta el quintil de ingreso aumentan la cantidad de horas de trabajo remunerado y disminuyen las de trabajo no remunerado (CEPAL, 2009: 47). Ello implica que las mujeres de los quintiles más altos de ingreso encuentran en el mercado apoyo para la realización de las labores reproductivas del hogar, y de este modo pueden aumentar su participación en el mercado laboral. Sin embargo, las mujeres de los quintiles de ingreso inferiores no cuentan con los re- 
cursos para hacerlo. En ninguno de los dos escenarios los hombres aumentan su tiempo de trabajo no remunerado en el hogar.

Como en México la pareja genera un efecto de dependencia económica en las mujeres porque al encontrarse en esta situación es menor su probabilidad de participar en el mercado laboral, el efecto de la familia en la pobreza femenina puede ser mixto. Por un lado muchas mujeres son pobres aunque no vivan en hogares pobres debido a la desigual distribución de los recursos. El cuadro 2 muestra cómo bajo el modelo colectivo, las mujeres que viven en hogares encabezados por hombres tienen una tasa de pobreza de $51 \%$, mientras la de los hombres en este mismo tipo de hogar es de $34 \%$. Pero también se da el caso contrario: las mujeres pueden escapar de la pobreza gracias a los recursos compartidos en el hogar, a pesar de que no cuenten con sus propios recursos. Lo que sí es claro es que como las mujeres no cuentan con transferencias gubernamentales, cuando no participan en el mercado laboral, su dependencia del ingreso de la pareja las pone en una situación de vulnerabilidad que disminuye su capacidad de decisión en el interior del hogar y las puede inhabilitar para abandonar el hogar en caso de que lo necesitara. El caso contrario se observa en los hogares encabezados por mujeres, quienes cuentan con fuentes de ingreso propias, ya sea porque participan en el mercado laboral o porque reciben transferencias (Villareal y Shin, 2008).

\section{Conclusiones}

El primer objetivo de este trabajo ha sido mostrar evidencias de la pobreza femenina utilizando el modelo colectivo de hogar con vistas a lograr un cálculo más realista sobre los recursos a los cuales ellas pueden tener acceso y evitar la crítica más común al análisis de la pobreza femenina. Si bien se trata de un ejercicio aproximativo, dado que no se conoce con exactitud la cantidad de recursos que son retenidos por los miembros del hogar para su propio consumo y porque se usa la Línea de Bienestar de Coneval -cuestionable por la manera en que calcula la pobreza- resulta evidente que asumiendo solamente que la retención constituye una pequeña cantidad $(20 \%)$ y lo demás es puesto en la bolsa común del hogar, las mujeres enfrentan desventajas debido a su menor acceso a fuentes de ingreso independientes. Las brechas de género en la pobreza se sostienen incluso si se toman en cuenta los costos de generar el ingreso. Se constata que a igual si- 
tuación en términos de otras divisiones sociales -como el lugar de residencia urbano o rural, la pertenencia o no a un grupo indígena, la edad, el nivel educacional y la situación de inactividad-, la condición de mujer suma desventajas adicionales en el acceso a recursos. La excepción a lo anterior solamente se presenta cuando está empleada o tiene un alto nivel educacional (posgrado). En esos casos su situación es idéntica o ligeramente mejor que la de su contraparte masculina.

El segundo objetivo de este artículo ha sido explorar el peso de los distintos procesos por los cuales las mujeres se ven limitadas en la sociedad para obtener recursos, ya que conocer una situación es el primer paso para que ésta pueda ser modificada. Se encuentra que el estatus ocupacional es el primer factor en orden de importancia que incide en las posibilidades de que la mujer pueda acceder o no a mayores recursos propios; si participa en el mercado laboral formal sus probabilidades de pobreza son las más bajas, y en el otro extremo, si es inactiva sus posibilidades de pobreza son extremadamente altas. En segundo orden figura su nivel educacional. Si su educación es de posgrado sus posibilidades de pobreza son prácticamente inexistentes, y éstas van aumentando a medida que disminuye el nivel educacional: si es analfabeta sus probabilidades de pobreza son elevadísimas. Posteriormente se advierte que contar con acceso a la seguridad social disminuye notablemente las probabilidades de estar en pobreza, y este efecto es aún más fuerte para las personas de la tercera edad. Asimismo el papel de la familia funciona claramente elevando las posibilidades de estar en pobreza. Una mujer sola es menos pobre que si tiene pareja, y notablemente menos pobre que si tiene hijos. Por último, se encuentra que la condición de etnicidad también provoca un menor acceso a los recursos.

La importancia del trabajo para disminuir la pobreza resulta más o menos obvia debido a que genera recursos propios y acceso a la seguridad social, además de que puede traer ventajas adicionales como contribuir a ampliar el capital social femenino y su seguridad e independencia. La incorporación al mercado laboral no sólo beneficia la situación de las mujeres en edad activa, sino que genera un efecto positivo para cuando llegan a la tercera edad porque les garantiza una pensión. Sin embargo, la incorporación femenina al mercado laboral resulta sumamente baja en comparación con la de su contraparte masculina.

No obstante, la manera en que actúa la política social también se perfila como una fuente importante de la pobreza femenina en México. Para quienes no pueden participar en el mercado laboral, una de 
las maneras más efectivas de evitar la pobreza es por medio de la política social. Como se vio, las mujeres que son más pobres debido a las desigualdades que enfrentan en el interior de los hogares encuentran mayores dificultades para participar en el mercado laboral debido a sus labores reproductivas, y esto genera un círculo vicioso en el cual se vuelve prácticamente imposible que puedan trascender su pobreza. En este caso la política social no compensa tal situación proveyendo recursos de apoyo. Cuando las políticas protegen el derecho de las madres a un ingreso independiente, éstas se encuentran en una mejor situación, como se demuestra en el caso de los hogares encabezados por mujeres. Muchos de estos hogares cuentan con transferencias en forma de pensión alimentaria (por divorcio) o de pensión de la seguridad social (por viudez).

Otro aspecto fundamental en que la política social podría mejorar la situación de la mujer para acceder a ingresos es la procuración de servicios públicos universales como guarderías o servicios de cuidado para otros miembros del hogar, de modo que ayude a disminuir la carga femenina de trabajo no remunerado en el hogar. Igualmente es necesario buscar los mecanismos para que las mujeres puedan acceder a mayores niveles educacionales, ya que se ha comprobado la importancia de la educación para evitar la pobreza. Asimismo es preciso garantizar una pensión universal para las mujeres de la tercera edad que no cuentan con ella, ya que su importancia es crucial para disminuir la pobreza en esta etapa de la vida.

Si bien es fundamental mejorar las fuentes de ingreso femeninas, esto por sí solo no resulta suficiente. Como se evidencia en este estudio, entre las principales causas de la pobreza femenina destacan las desigualdades en la esfera doméstica, y esto no se resuelve solamente al mejorar su situación material (García y Oliveira 1994; González de la Rocha, 1994; Johnsson-Latham, 2004; Johnson, 2005); se requiere también realizar acciones más amplias que busquen lograr la equidad social de las mujeres, particularmente en el interior de sus hogares. Sin embargo, la mayoría de las políticas se han centrado solamente en lograr su participación en el mercado laboral, sin tomar en cuenta que la principal razón por la cual no participan y viven en una situación de subordinación económica es por su carga de trabajo no remunerado (CEPAL, 2009: 26). Por tanto, las políticas dirigidas a atacar la desigualdad de género y que promuevan una mayor equidad en la división sexual del trabajo resultarán sumamente positivas para mejorar la situación de pobreza de las mujeres. 
Por último, es importante mencionar la necesidad de contar con fuentes de información que permitan conocer la manera en que los recursos se distribuyen en el interior de los hogares, de modo que se cuenten con datos más precisos para seguir investigando sobre este problema.

\section{Bibliografía}

Boltvinik, Julio (2001), “Opciones metodológicas para medir la pobreza en México”, Comercio Exterior, vol. 51, núm. 11, pp. 869-878.

Boltvinik, Julio (2003), "Welfare, Inequality and Poverty in Mexico, 1970-2000", en Kevin Middlebrook y Eduardo Zepeda (coords.), Confronting Development: Assessing Mexico Economic and Social Policy Challenges, Stanford, Stanford University Press, pp. 385-446.

Boltvinik, Julio (2009), "Economía moral: Coneval, nuevo método de medición baja la pobreza”, La Jornada, México, 11 de diciembre.

Boltvinik, Julio (2012), "Economía moral: A 30 años del estudio de Coplamar sobre necesidades esenciales / VII, Costo de alimentarse fuera del hogar. Estudio de COA para Evalúa DF", La Jornada, México, 23 de noviembre.

Burchardt, Tania, Julian Le Grand y David Piachaud (2002), "Introduction", en John Hills, Julian Le Grand y David Piachaud (coords.), Understanding Social Exclusion, Oxford, Oxford University Press, pp. 1-12.

Chant, Sylvia (1997), "Women-Headed Households: Poorest of the Poor? Perspectives from Mexico, Costa Rica and the Philippines", IDS Bulletin, vol. 28, núm. 3, pp. 26-48.

Chant, Sylvia (1998), "Mitos y realidades de la formación de las familias encabezadas por mujeres: el caso de Querétaro, México”, en Luisa Gabayet, Patricia García, Mercedes González, Silvia Lailson y Agustín Escobar (comps.), Mujeres y sociedad. Salarios, hogar y acción social en el occidente de México, México, El Colegio de Jalisco / CIESAS, pp. 181-203.

Chant, Sylvia (2007), Gender, Generation and Poverty: Exploring the "Feminisation of Poverty" in Africa, Asia and Latin America, Cheltenham, Edward Elgar.

Chant, Sylvia (2009), "The 'Feminisation of Poverty' in Costa Rica: To What Extent a Conundrum?”, Bulletin of Latin American Research, vol. 28, núm. 1, pp. 19-43.

Chant, Sylvia (2010), "Gendered Poverty across Space and Time: Introduction and Overview", en Sylvia Chant (coord.), The International Handbook of Gender and Poverty. Concepts, Research and Policy, Cheltenham, Edward Elgar, pp. 1-28.

CEPAL (2004), Roads toward Gender Equity in Latin American and the Caribbean, Santiago de Chile, Comisión Económica para América Latina y el Caribe. CEPAl (2008), Anuario Estadístico de América Latina y el Caribe, Santiago de Chile, Comisión Económica para América Latina y el Caribe. 
CEPAl (2009), Panorama social de América Latina, Santiago de Chile, Comisión Económica para América Latina y el Caribe.

Coneval (2008), Informe de evaluación de la política de desarrollo social en México: 2008, México, Consejo Nacional de Evaluación de la Política de Desarrollo Social.

Coneval (2009), Metodología para la medición multidimensional de la pobreza en México, México, Consejo Nacional de Evaluación de la Política de Desarrollo Social.

Costa, Joana y Elydia Silva (2010), "Gender Inequalities and Poverty: A Simulation of the Likely Impacts of Reducing Labour Market Inequalities on Poverty Incidence in Latin America”, en Sylvia Chant (coord.), The International Handbook of Gender and Poverty. Concepts, Research and Policy, Cheltenham, Edward Elgar, pp. 490-496.

Damián, Araceli (2003), "Tendencias recientes de la pobreza con enfoque de género en América Latina”, Papeles de Población, núm. 38, pp. 27-76.

Damián, Araceli (2008), "Clase de hogar y pobreza”, ElFinanciero, 22 de diciembre. Damián, Araceli (2011), "Pobreza por jefatura y edad”, El Financiero, 25 de abril.

Evalúa DF (2011), CNA-DF, Canasta normativa alimentaria para el Distrito Federal, México, Consejo de Evaluación del Desarrollo Social del Distrito Federal $<$ www.evaluadf.gob.mx $>$.

Falkingham, Jane y Angela Baschieri (2009), "Gender and Poverty: How Misleading is the Unitary Model of Household Resources? An Illustration from Tajikistan”, Global Social Policy, vol. 9, núm. 43, pp. 43-62.

Falkingham, Jane y Angela Baschieri (2010), "Gender, Poverty and Transition in Central Asia”, en Sylvia Chant (coord.), The International Handbook of Gender and Poverty. Concepts, Research and Policy, Cheltenham, Edward Elgar, pp. 184-189.

García Brígida y Orlandina de Oliveira (1994), Trabajo femenino y vida familiar en México, México, El Colegio de México.

González de la Rocha, Mercedes (1994), The Resources of Poverty. Women and Survival in a Mexican City, Oxford, Blackwell.

Graham, Carol (1996), "Gender Issues in Poverty Alleviation: Recent Experiences with Demand-Based Programmes in Latin American, Africa and Eastern Europe", documento de trabajo, Ginebra, Organización Internacional del Trabajo (Issues in Development Discussion Paper).

Heintz, James (2010), "Women's Employment, Economic Risk and Poverty" en Sylvia Chant (coord.), The International Handbook of Gender and Poverty. Concepts, Research and Policy, Cheltenham, Edward Elgar, pp. 434-439.

INEGI (2008), Encuesta Nacional de Ingreso y Gasto de los Hogares 2008, Aguascalientes, Instituto Nacional de Estadística y Geografía.

Johnson, Robert (2005), "Not a Sufficient Condition: The Limited Relevance of the Gender MDG to Women's Progress", en Caroline Sweetman (coord.), Gender and the Millenium Development Goals, Oxford, Oxfam, pp. 56-66. 
Johnsson-Latham, Gerd (2004), "Understanding Female and Male Poverty and Deprivation", en Gerd Johnsson-Latham (coord.), Power and Privileges: Gender Discrimination and Poverty, Estocolmo, Gobierno de Suecia, pp. 16-45.

Lister, Ruth (2010), “Linking Women's and Children's Poverty”, en Sylvia Chant (coord.), The International Handbook of Gender and Poverty. Concepts, Research and Policy, Cheltenham, Edward Elgar, pp. 167-172.

Marshall, Thomas H. (1950), Citizenship and Social Class and Other Essays, Cambridge, Cambridge University Press.

Medeiros, Marcelo y Joana Costa (2010), "The Feminisation of Poverty: A Widespread Phenomenon?”, en Sylvia Chant (coord.), The International Handbook of Gender and Poverty. Concepts, Research and Policy, Cheltenham, Edward Elgar, pp. 95-100.

Moghadam, Valentin (1997), "The Feminisation of Poverty: Notes on a Concept and Trend”, Women Studies Occasional Paper, núm.2, Normal, Illinois State University.

Molyneux, Maxine (2006a), "Mothers at the Service of the New Poverty Agenda: Progresa / Oportunidades, Mexico's Conditional Transfer Programmme", Journal of Social Policy and Administration, vol. 40, núm. 4, pp. 425-49.

Molyneux, Maxine (2006b), "Poverty Relief and the New Social Policy in Latin America: Mothers at the Service of the State?", document de trabajo, Ginebra, United Nations Research Institute for Social Development.

Nauckhooff, Eva (2004), "Poverty without the Poor", en Gerd Johnsson-Latham (coord.), Power and Privileges: Gender Discrimination and Poverty, Estocolmo, Gobierno de Suecia, pp. 46-73.

Nolan, Brian y Christopher Whelan (1996), Resources, Deprivation and Poverty, Oxford, Clarendon Press.

Ordorica, Manuel (2010), "Las proyecciones de población hasta la mitad del siglo XXI”, en Brígida García y Manuel Ordorica (coords.), Los grandes problemas de México, tomo 1, "Población”, México, El Colegio de México, pp. 29-51.

Pahl, Jan (1989), Money and Marriage, Londres, Macmillan.

Payne, Geoff (2006), "Social Divisions as a Sociological Perspective”, en Geoff Payne (coord.), Social Divisions, Nueva York, Palgrave Macmillan, pp. 3-22.

Perrons, Diane (2010), "Gender, Work and Poverty in High-Income Countries", en Sylvia Chant (coord.), The International Handbook of Gender and Poverty. Concepts, Research and Policy, Cheltenham, Edward Elgar, pp. 409-414.

Quisumbing, Agnes R. (2010), "Gender and Household Decision-Making in Developing Countries: A Review of Evidence", en Sylvia Chant (coord.), The International Handbook of Gender and Poverty. Concepts, Research and Policy, Cheltenham, Edward Elgar, pp. 161-166.

Razavi, Shahra y Silke Staab (2010), "Gender, Poverty and Inequality: The Role of Markets, States and Households", en Sylvia Chant (coord.), The Inter- 
national Handbook of Gender and Poverty. Concepts, Research and Policy, Cheltenham, Edward Elgar, pp. 427-433.

Rodríguez, Katya (2010), Poverty in Mexico at the Beginning of the Twenty-First Century: An Alternative Analysis, Saarbrücken, Lambert Academic Publishing.

Rodríguez, Katya (2011), "Pobreza y política social en México 2000-2010: ¿una década de cambio?, en Mónica Uribe (coord.), Los vaivenes de las políticas sociales en América Latina Argentina, Colombia, Chile, México y Uruguay: ¿¿Neo o Posneoliberalismo?, México, Porrúa, pp. 139-178.

Rodríguez, Katya (2012), “¿Existe feminización de la pobreza en México? La evidencia bajo un cambio del modelo unitario al modelo colectivo de hogar", Papeles de Población, vol. 18, núm. 72, pp. 181-2012.

Sedesol (2002), "Medición de la pobreza: variantes metodológicas y estimación preliminar", documentos de investigación, México, Secretaría de Desarrollo Social.

Sen, Gita (2010), "Poor Households or Poor Women: Is There a Difference?", en Sylvia Chant (coord.), The International Handbook of Gender and Poverty. Concepts, Research and Policy, Cheltenham, Edward Elgar, pp. 101-104.

Townsend, Peter (1979), Poverty in the United Kingdom, Londres, Allen Lane and Penguin Books.

Villareal, Andrés y Heeju Shin (2008), "Unraveling the Economic Paradox of Female-Headed Households in Mexico: The Role of Family Networks", The Sociological Quarterly, vol. 49, pp. 565-595.

\section{Acerca de la autora}

Katya Rodríguez Gómez es doctora en sociología por la Universidad de Essex, maestra en Estudios Urbanos por El Colegio de México y licenciada en Sociología por la Universidad de La Habana. Sus principales líneas de investigación son pobreza, género y política social. Pertenece al Sistema Nacional de Investigadores. Actualmente es profesora investigadora de la División de Ciencias Sociales y Humanidades de la Universidad de Guanajuato, campus León. 